Article

\title{
Air Contaminants and Atmospheric Black Carbon Association with White Sky Albedo at Hindukush Karakorum and Himalaya Glaciers
}

\author{
Irfan Zainab ${ }^{1}$, Zulfiqar Ali ${ }^{1, *(D)}$, Usman Ahmad ${ }^{2}$, Syed Turab Raza ${ }^{3, *}$, Rida Ahmad ${ }^{1}$, Zaidi Zona ${ }^{1}$ \\ and Safdar Sidra 4
}

check for

updates

Citation: Zainab, I.; Ali, Z.; Ahmad, U.; Raza, S.T.; Ahmad, R.; Zona, Z.; Sidra, S. Air Contaminants and Atmospheric Black Carbon Association with White Sky Albedo at Hindukush Karakorum and Himalaya Glaciers. Appl. Sci. 2022, 12, 962. https://doi.org/10.3390/ app12030962

Academic Editors: Yves Rybarczyk and Rasa Zalakeviciute

Received: 22 December 2021

Accepted: 11 January 2022

Published: 18 January 2022

Publisher's Note: MDPI stays neutral with regard to jurisdictional claims in published maps and institutional affiliations.

Copyright: (c) 2022 by the authors. Licensee MDPI, Basel, Switzerland. This article is an open access article distributed under the terms and conditions of the Creative Commons Attribution (CC BY) license (https:// creativecommons.org/licenses/by/ $4.0 /)$.
1 Environmental Health and Wildlife Laboratory, Institute of Zoology, University of the Punjab, Lahore 54000, Pakistan; zainabirfan90@gmail.com (I.Z.); ridaahmad411@gmail.com (R.A.); zaidizona1@gmail.com (Z.Z.)

2 Center of Earth and Environmental Sciences, University of the Punjab, Lahore 54000, Pakistan; usmanpassion@gmail.com

3 Yunnan Key Laboratory of Plant Reproductive Adaptation and Evolutionary Ecology, Yunnan University, Kunming 650500, China

4 Department of Ecology and Wildlife, University of Veterinary and Animal Sciences, Lahore 54000, Pakistan; sidra.safdar@uvas.edu.pk

* Correspondence: zali.zool@pu.edu.pk (Z.A.); s.turabkazmi@imde.ac.cn (S.T.R.)

\begin{abstract}
Environmental contaminants are becoming a growing issue due to their effects on the cryosphere and their impact on the ecosystem. Mountain glaciers are receding in the HKH region and are anticipated to diminish further as black carbon $(\mathrm{BC})$ concentrations rise along with other pollutants in the air, increasing global warming. Air contaminants and BC concentrations were estimated (June 2017-May 2018). An inventory of different pollutants at three glaciers in Karakoram, Hindukush, and the Himalayas has been recorded with Aeroqual 500 and TSI DRX 8533, which are as follows: ozone $\left(28.14 \pm 3.58 \mu \mathrm{g} / \mathrm{m}^{3}\right)$, carbon dioxide $\left(208.58 \pm 31.40 \mu \mathrm{g} / \mathrm{m}^{3}\right)$, sulfur dioxide $\left(1.73 \pm 0.33 \mu \mathrm{g} / \mathrm{m}^{3}\right)$, nitrogen dioxide $\left(2.84 \pm 0.37 \mu \mathrm{g} / \mathrm{m}^{3}\right), \mathrm{PM}_{2.5}\left(15.90 \pm 3.32 \mu \mathrm{g} / \mathrm{m}^{3}\right), \mathrm{PM}_{10}\left(28.05 \pm 2.88 \mu \mathrm{g} / \mathrm{m}^{3}\right)$, total suspended particles $\left(76.05 \pm 10.19 \mu \mathrm{g} / \mathrm{m}^{3}\right)$, BC in river water $\left(88.74 \pm 19.16 \mu \mathrm{g} / \mathrm{m}^{3}\right)$, glaciers $\left(17.66 \pm 0.82 \mu \mathrm{g} / \mathrm{m}^{3}\right)$, snow $/$ rain $(57.43 \pm 19.66 \mathrm{ng} / \mathrm{g})$, and air $\left(2.80 \pm 1.20 \mu \mathrm{g} / \mathrm{m}^{3}\right) . \mathrm{BC}$ was estimated by using DRI Model 2015, Multi-Wavelength Thermal/Optical Carbon Analyzer, in conjunction with satellite-based white-sky albedo (WSA). The average BC concentrations in the Karakoram, Himalaya, and Hindukush were $2.35 \pm 0.94,4.38 \pm 1.35$, and $3.32 \pm 1.09\left(\mu \mathrm{g} / \mathrm{m}^{3}\right)$, whereas WSA was $0.053 \pm 0.024,0.045 \pm 0.015$, and $0.045 \pm 0.019\left(\mu \mathrm{g} / \mathrm{m}^{3}\right)$, respectively. Regression analysis revealed the inverse relationship between WSA and BC. The resulting curves provide a better understanding of the non-empirical link between BC and WSA. Increased BC will inherit ecological consequences for the region, ultimately resulting in biodiversity loss.
\end{abstract}

Keywords: black carbon; white-sky albedo; air contaminants; HKH region Pakistan; glaciers; regression

\section{Introduction}

The Hindukush-Karakoram-Himalaya $(\mathrm{HKH})$ region is frequently called the "water towers of Asia" and are at the forefront of the change in the climate. Furthermore, from the beginning of the 1990s, global glacier loss might be as high as $70 \%$ due to anthropogenic sources [1]. Bolch et al. [2] indicated that despite the spatial differences, the loss of the $\mathrm{HKH}$ glacier mass appears to be related or somewhat lower than that of other glacial areas. Generally, monsoon in the southeast and westerlies in the northwest, the mass balance patterns from the southeast to the northwest of $\mathrm{HKH}$ are contrasted. Nevertheless, the processes affecting Himalayan glaciers are still not well known. Brown et al. [3] suggested that the variations in $\mathrm{HKH}$ glacial mass impacting main rivers flow in the $\mathrm{HKH}$ that ultimately support more than 1.3 billion inhabitants. The HKH comprises around 
52,000 glaciers and covers an area of approximately $60,000 \mathrm{~km}^{2}$. The glacier altitude ranges from 2409 mean above sea level (masl). The highest seasonal snowpack in the HKH area can surpass 1.79 million $\mathrm{km}^{2}$, or $42.9 \%$ of the entire area of land [4].

The atmospheric temperature has a rise of approximately between 0.8 and $1.2{ }^{\circ} \mathrm{C}$ due to greenhouse gases [5] and the world is at the edge of reaching the threshold of hazardous climate change which implies that study of all possible agents and pollutants is crucial. Apart from long-lived gases, short-lived agents including tropospheric ozone and black carbon are also responsible for climate warming [6]. Tropospheric ozone is formed by the reaction of nitrous oxides and volatile organic compounds which are released from fossil fuels and organic chemicals, respectively [7]. The BC influence on the cryosphere has garnered a lot of interest, according to Ramanathan and Carmichael [8], BC can accelerate melting through a variety of mechanisms, including climatic warming that lower albedo due to its deposition on snow surfaces. The HKH is located close to some of the most significant sources of black carbon, and the proximity to identified transit routes makes it particularly vulnerable [9].

According to the World Resources Institute (WRI), black carbon is the second most significant climate pollutant after carbon dioxide, with total annual global emissions of $7500 \mathrm{Gg} \mathrm{yr}^{-1}$ (with an uncertainty range of 2000-29,000 $\mathrm{Gg} \mathrm{yr}^{-1}$ ) and total annual global climate forcing of approximately $1.1 \mathrm{~W} \mathrm{~m}^{-2}$ (high uncertainty range $0.17-2.1 \mathrm{~W} \mathrm{~m}^{-2}$ ) [5]. Black carbon is a unique carbonaceous material that greatly absorbs forthcoming solar radiations and is produced primarily through incomplete combustion of fuels such as petroleum, biomass, coal, and biofuels. BC is extensively present in the atmosphere, including snow, rain, air, soil, and sediments [10]. BC concentration in the air is the main driver of rapid glacial retreat and major climatic radiative forcing along with the decrease in participation and rise in temperature. BC is mainly characterized by the absorbance of solar radiation and resistance to chemical modification. In the atmosphere, black carbon does not let the trapped solar energy emit back. Consequently, that raises the temperature and timing of glacial runoff. Black carbon has potentially strong impacts on snow cover and glacier melt that leads to positive climate forcing. By depositing onto the snow and ice surface, BC darkens the surface of glaciers and snowpacks by reducing their albedo [10]. However, there is a noticeable lack of field measurements concerning spatial and temporal distribution of $\mathrm{BC}$ on inland glaciers of HKH Pakistan with the degree of change in albedo caused by the BC that leads to the accelerated snow and glacier melt.

The complexity and susceptibility of mountain habitats to climate change are well understood [11,12]. HKH are among the mountain systems designated as "critical zones" [12,13]. Due to their high altitude and variable debris cover, alpine glaciers in the HKH are regarded to be particularly vulnerable to climate change [14]. Furthermore, climate forcing is assumed to be a direct cause of such high-altitude geodynamic systems $[15,16]$. To resolve these glaciological snags, it is necessary to have a fundamental understanding of the feedbacks that exist between climatic forcing and glacier response [17]. This necessitates thorough information on glacier studies and ice volumes, mass-balance gradients, regional mass-balance trends, landscape features, and pollutants such as BC in the air, which can affect albedo and regulate ablation and require consideration.

Few studies have already focused on observations and characterization of BC particles in the region. However, in HKH Pakistan $[2,9,10,18,19]$, current concentrations of BC and albedo were yet to be estimated to find out their possible relationship. Therefore, aiming to have a better understanding of $\mathrm{BC}$ concentration in relation to the albedo reduction in different seasons was assumed to be important.

\section{Materials and Methods}

\subsection{Study Site}

Pakistan has some of the world's largest and longest mid-latitude glaciers due to their numerous high mountains and ample precipitation typical of a monsoon climate. Glacierized land is estimated to occupy $15,000 \mathrm{~km}^{2}$ (or $37 \%$ ) of the high mountains [20]. 
According to the Intergovernmental Panel on Climate Change report, glaciers in the HKH are melting rapidly [21]. Pakistan has 7253 recognized glaciers [22] and almost all of them are in Gilgit-Baltistan and Khyber Pakhtunkhwa's northern areas. The global average mass balance of glaciers is indisputably negative [23]. The majority of Himalayan glaciers are losing mass at rates comparable to those seen elsewhere [2,24].

The sites for the current study include three mountain ranges, namely the Himalaya, Hindukush, and Karakoram ranges. These three sites were chosen to investigate black carbon concentrations in relation to solar radiation reflection (albedo) Figure 1.

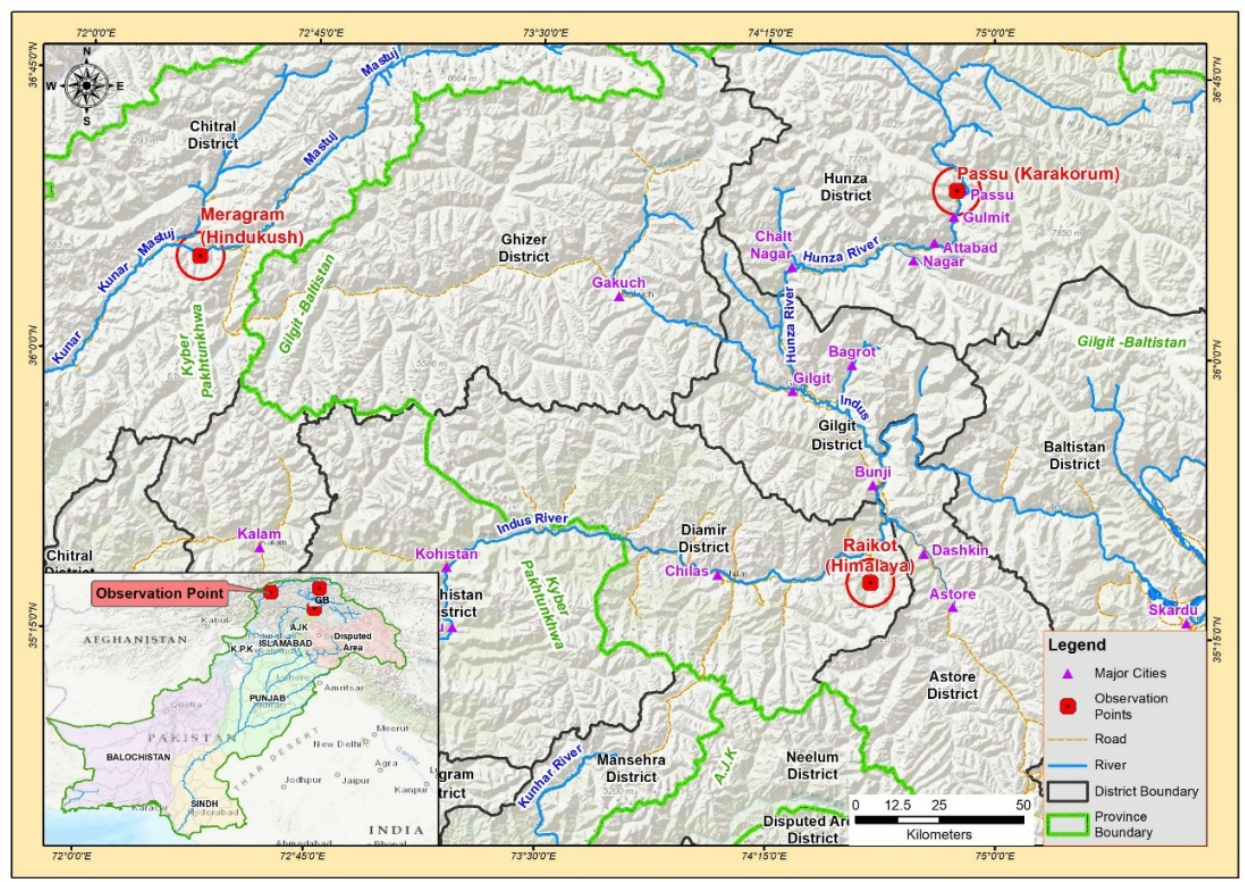

Figure 1. The study area of observational sites.

The Karakoram Mountain glaciers account for roughly 3\% of the total in recent times [2,23]. The Passu glacier is located south of the Batura glacier, between $36^{\circ} 27^{\prime} \mathrm{N}$ and $36^{\circ} 28^{\prime} \mathrm{N}$, and $74^{\circ} 38^{\prime} \mathrm{E}$ and $74^{\circ} 52^{\prime} \mathrm{E}$. It feeds the Hunza River, which flows west to east through northern Pakistan [25]. The sampling site in Passu glacier is located at $74^{\circ} 52^{\prime} 26.94^{\prime \prime} \mathrm{E}$ and $36^{\circ} 27^{\prime} 20.10^{\prime \prime} \mathrm{N}$ at the elevation of 2640 masl (Figure 1). It was discovered that a large volume of water was trapped beneath the ice near the terminus (tongue) of numerous glaciers, including the Passu glacier, which had already caused outbursts. As a result, the massive volume of water that was constantly expanding was not visible, causing catastrophic damage downstream to human lives, towns, and infrastructure [18].

The Hindukush Range stretches from the Pamir Plateau's western boundary to the Karakoram's western edge. Pakistan, Afghanistan, and China are all separated by it. It contains snow-covered mountains that are traversed by a number of glaciers. Noshak (7369 masl) and Tirich Mir (7690 masl) are the highest peaks in the area. The mountain range is drained into Chitral, Kunar, Punjkora, and Swat rivers [16]. The sampling site in the Meragram glacier is located at $72^{\circ} 22^{\prime} 7.56^{\prime \prime} \mathrm{E}$ and $36^{\circ} 15^{\prime} 11.94^{\prime \prime} \mathrm{N}$ at the elevation of 2345 masl (Figure 1).

The Nanga Parbat massif is the high Himalaya's northwestern limit with the highest height variation ranging from 1030 masl in the Indus Gorge to 8126 masl at the peak of Nanga Parbat. Raikot Glacier, on Nanga Parbat's north flank, is about $15 \mathrm{~km}$ long and covers $39 \mathrm{Km}^{2}$. Through substantial fluctuations in the steepness of the glacier profile, the ice tongue drops to 3180 masl [26]. The sampling site in the Raikot glacier is located at $74^{\circ} 35^{\prime} 31.38^{\prime \prime} \mathrm{E}$ and $35^{\circ} 24^{\prime} 18.48^{\prime \prime} \mathrm{N}$ at the elevation of 2683 masl (Figure 1). 


\subsection{Temperature, Precipitation, and Humidity}

The average temperature in Hindukush, Karakoram, and Himalaya was 9.52, 9.28, and $12.05\left({ }^{\circ} \mathrm{C}\right)$, respectively. The highest recorded temperature was $19.71{ }^{\circ} \mathrm{C}$ in July 2017 and the lowest was $2.92{ }^{\circ} \mathrm{C}$ in January 2018 as shown in Figure 2a. The humidity of the three sites is shown in Figure $2 b$.

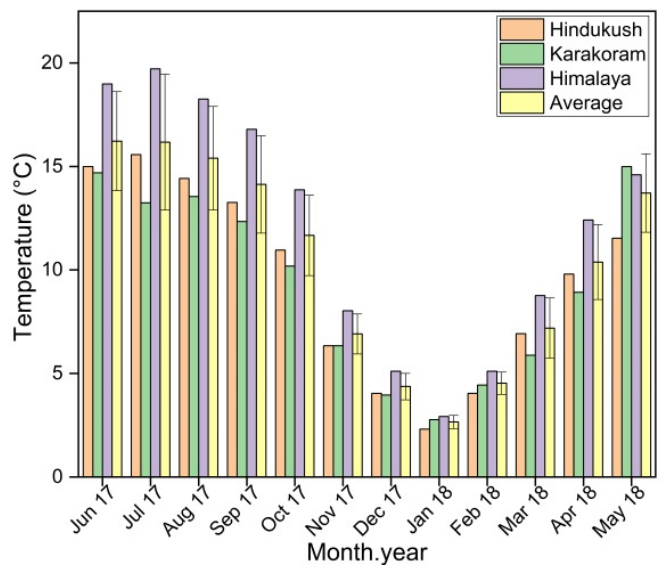

(a)

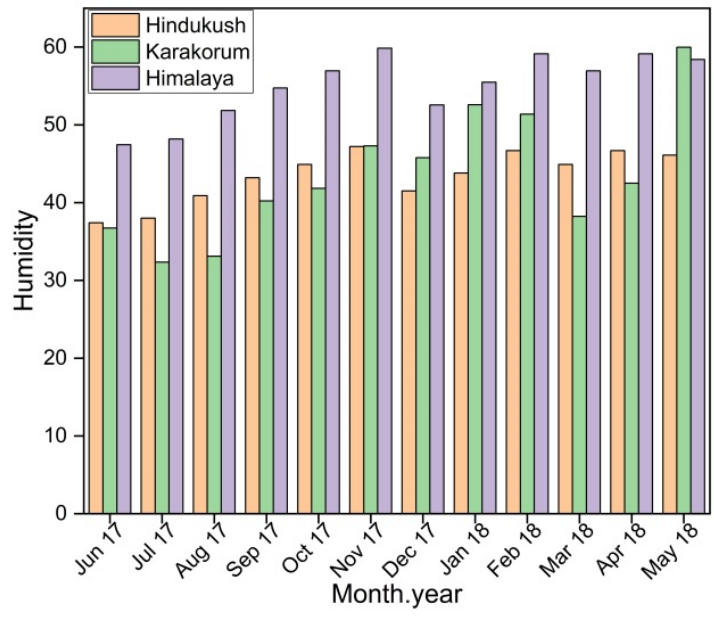

(b)

Figure 2. Comparative Temperature (a) and humidity (b) variation in Hindukush, Karakorum, and Himalaya.

The average precipitation in Hindukush, Karakoram, and Himalaya was 19.78, 6.43 and $10.55(\mathrm{~mm})$, respectively. The maximum precipitation $(60.2 \mathrm{~mm})$ was observed in March 2018 at Hindukush as shown in Figure 3.

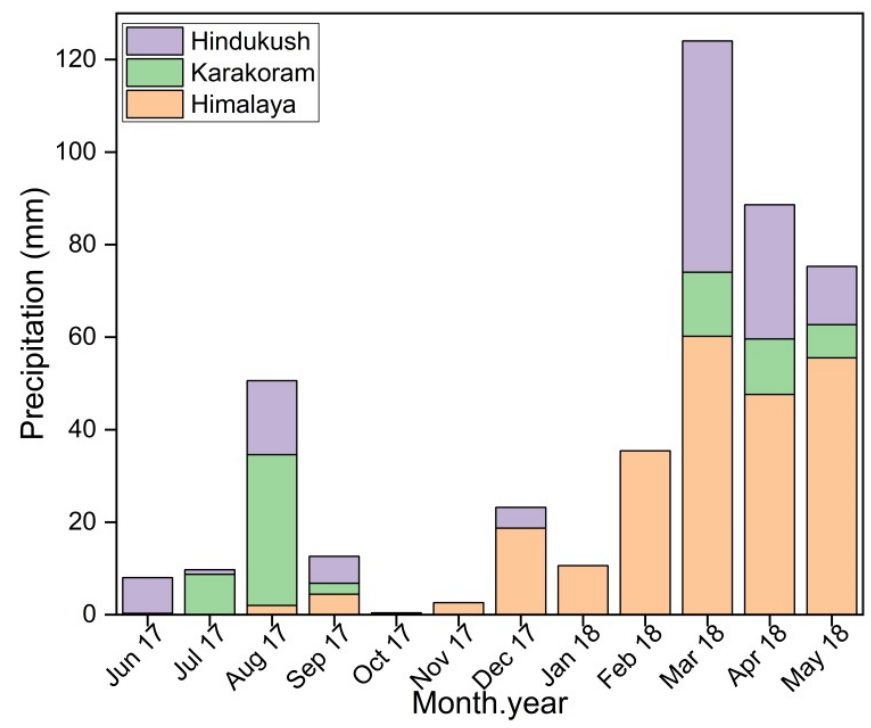

Figure 3. Comparative precipitation variation in Hindukush, Karakorum, and Himalaya.

\subsection{Work Methodology}

To estimate the ambient $\mathrm{BC}$ in the atmospheric zone, real-time measurement of black carbon mass concentration was conducted for six hours, once a month from June 2017 to May 2018. Air samples were collected using a volumetric sampler at a flow rate of $35 \mathrm{~L} / \mathrm{min}$ on quartz microfiber filters (WhatmanTM 1851-047 Grade QM-A Quartz Microfiber Filter for Air Sampling, Diameter $4.7 \mathrm{~cm}$ Filters, Pore Size: $2.2 \mu \mathrm{m}$ ) and were sent to the Institute of Tibetan Plateau Research, Chinese Academy of Sciences for analysis. 
To the real-time particulate matter data directly from the air, Dust Trak DRX Aerosol Monitor 8533 was used. The outdoor metrological parameters were monitored using Kestral Weather Station while Aeroqual 500 series air quality sensor was used to monitor gas emissions including ozone $\left(\mathrm{O}_{3}\right)$, carbon dioxide $\left(\mathrm{CO}_{2}\right)$, sulfur dioxide $\left(\mathrm{SO}_{2}\right)$, and nitrogen dioxide $\left(\mathrm{NO}_{2}\right)$. The DRI Model 2015 (Multi-Wavelength Thermal/Optical Carbon Analyzer) was used to measure organic carbon (OC), elemental carbon (EC) (also known as black carbon), and temperature-separated carbon fractions on aerosol filter deposits. It has a measurement range of 0.05 to $750 \mu \mathrm{g}$ carbon $/ \mathrm{cm}^{2}$, with a minimum detection limit of $0.43 \mu \mathrm{g} / \mathrm{cm}^{2}$ for OC and $0.12 \mu \mathrm{g} / \mathrm{cm}^{2}$ for EC. The methodology of Zhang et al. [27] was adopted for the current study.

\subsection{White-Sky Albedo}

This study calls for the use of the WSA 16-Day L3 Global 0.05 ${ }^{\circ}$ CMG MCD43C3 Modis product, which has the best quality assurance values. MCD43C3 Collection 6 datasets, which were produced daily with a 16-day accumulation window at $0.05^{\circ}$ spatial resolution combinations, were used to extract MODIS shortwave white-sky albedo products $\left(0.05^{\circ}\right.$ resolution is approximately $5 \mathrm{~km} \times 5 \mathrm{~km}$ on ground). Following that, MODIS Albedo data were obtained, processed, and analyzed to determine its link with black carbon.

The United States Geological Survey (USGS) Earth Explorer website (https: / / earthexplorer. usgs.gov, accessed on 8 July 2020) has been used to retrieve MODIS MCD43C3 albedo data. Data comprised of 365 days, starting from 1 June 2017, to 31 May 2018, in the form of hierarchical data format (HDF) files. Each HDF file is further comprised of 25 Scientific Datasets (SDS) layers among which 10 layers of BSA, 10 layers of WSA, and five layers of BRDF Albedo qualitative parameters. WSA data of only sampling dates have been processed to establish an association with BC concentrations at the sites. All the data was processed in ESRI ArcGIS software. Data processing mainly includes WSA data extraction from HDF files, clipping of data as per study area boundary, determination of WSA values from extracted data and tabular summarization along with some analysis using MS Excel.

\subsection{Data Analysis}

The relationship between the dependent variable and one or more independent variables is described using a linear regression model. All of the processes employed in a regression analysis, as well as the results reached, are based on the assumptions of the regression model. The centering predictor variable in the polynomial regression model is useful when there is reason to believe that the connection between two variables is curvilinear, and proposed centering as a technique of reducing multicollinearity.

The polynomial model is maintained as simple as possible in terms of the order. Arbitrary fitting of higher-order polynomials can be a major regression analysis miscalculation. A model that is consistent with data knowledge and its surroundings should be considered. It is always possible for a polynomial of order $(n-1)$ to pass through $\mathrm{n}$ locations, resulting in the discovery of a polynomial of sufficiently high degree that provides a "good fit" to the data. The $k$-th order polynomial model in one variable is given by

$$
y=\beta_{0}+\beta_{1} x+\beta_{2} x^{2}+\ldots+\beta_{k} x^{k}+\varepsilon
$$

If $x_{j}=x^{j}, j=1,2,3, \ldots, k$ then the model is multiple linear regressions model in $\mathrm{k}$ explanatory variables $x_{1}, x_{2}$, hen $x_{k}$. So, the linear regression model $y=X \beta+\varepsilon$ includes the polynomial regression model. As a result, the same approaches that are used to fit a linear regression model can also be used to fit a polynomial regression model. For example:

$$
y=\beta_{0}+\beta_{1} x+\beta_{2} x^{2}+\varepsilon
$$

or

$$
E(y)=\beta_{0}+\beta_{1} x+\beta_{2} x^{2}+\varepsilon
$$


This is a polynomial regression model with a single variable and called a second order regression model or quadratic model. The coefficients $\beta_{1}$ and $\beta_{2}$ denotes linear and quadratic effect parameters respectively.

Second-degree polynomial equation for regression analysis was used to establish and quantify the relationship between variables in a data set. Primary BC data and satellite WSA periodically summarized for three mountain ranges are summarized in Table 1. The resulting second-degree polynomial equations for three sampling sites at Karakoram, Himalaya, and Hindukush, along with cumulative data of BC and WSA using regression, are:

Table 1. Coefficient of determination values for three mountain ranges.

\begin{tabular}{cccc}
\hline $\begin{array}{c}\text { Hindukush } \\
\left(\mathbf{R}^{2}\right)\end{array}$ & $\begin{array}{c}\text { Karakoram } \\
\mathbf{( \mathbf { R } ^ { 2 } )}\end{array}$ & $\begin{array}{c}\text { Himalaya } \\
\mathbf{( \mathbf { R } ^ { 2 } )}\end{array}$ & $\begin{array}{c}\text { Cumulative } \\
\left(\mathbf{R}^{2}\right)\end{array}$ \\
\hline 0.5904 & 0.0082 & 0.5135 & 0.1575 \\
\hline
\end{tabular}

For Karakoram:

$$
y=-0.0023 x^{2}+0.0116 x+0.0406
$$

For Himalaya:

$$
y=-0.0054 x^{2}+0.0408 x-0.0219
$$

For Hindukush:

$$
y=-0.0077 x^{2}+0.0541 x-0.0329
$$

For Cumulative Data:

$$
y=-0.0043 x^{2}+0.0295 x+0.0052
$$

where $x$ represents $\mathrm{BC}$ and y represents WSA in the above equations as BC has been taken as an independent variable while WSA has been taken as a dependent variable. Coefficient of determination $\left(\mathrm{R}^{2}\right)$ of second order polynomial for regression are shown in the table below.

\subsection{Wind Plots}

For a better comprehension of BC data, it is necessary to determine wind drifts. BC in the air is a practice that varies in time and space according to how it moves, spreads, and is removed. As a result, knowledge of the procedure in various scales based on meteorological characteristics is required. The wind rose diagram provides a brief view of wind speed and path distribution at a specified location. Wind path is the representation of wind frequency using color bands interpreting wind speed ranges through a gridding polar coordinate system. Wind rose diagrams are developed to represent the wind blowing frequency from specific directions over a particular stage for three study sites (i.e., Hindukush, Karakoram, and Himalaya) for the sampling period (i.e., June 2017 to May 2018). Data for wind rose plots have been acquired from https:/ / worldweatheronline.com, accessed on 5 August 2020 [28]. Sixteen cordial directions have been used in wind roses such as north, north east, etc., even though they have thirty-two direction sub-divisions. As per measurement of angles (in degree), north, east, south and west correspond to $0^{\circ} / 360^{\circ}, 90^{\circ}, 180^{\circ}$ and $270^{\circ}$ respectively. Wind rose diagrams have been developed in WR plot view software. The distance end to end of every "spoke" about the loop is directly related to the frequency of wind blow from a specific direction per unit time. Every concentric circle signifies a dissimilar frequency, originating from zero at the middle to growing at the outer. Data to generate wind-rose plots have been summarized in Table 2. 
Table 2. Wind direction at study sites.

\begin{tabular}{|c|c|c|c|c|c|c|}
\hline \multirow{2}{*}{ Year } & \multicolumn{2}{|l|}{$\begin{array}{l}\text { Hindukush } \\
\text { (Meragram) }\end{array}$} & \multicolumn{2}{|c|}{$\begin{array}{c}\text { Karakoram } \\
\text { (Passu) }\end{array}$} & \multicolumn{2}{|c|}{$\begin{array}{c}\text { Himalaya } \\
\text { (Raikot) }\end{array}$} \\
\hline & $\begin{array}{l}\text { Wind Speed } \\
(\mathrm{m} / \mathrm{s})\end{array}$ & $\begin{array}{c}\text { Wind } \\
\text { Direction }\end{array}$ & $\begin{array}{c}\text { Wind Speed } \\
(\mathrm{m} / \mathrm{s})\end{array}$ & $\begin{array}{c}\text { Wind } \\
\text { Direction }\end{array}$ & $\begin{array}{l}\text { Wind Speed } \\
(\mathrm{m} / \mathrm{s})\end{array}$ & $\begin{array}{c}\text { Wind } \\
\text { Direction }\end{array}$ \\
\hline Jun-17 & 2.50 & NNW & 1.39 & NNW & 3.33 & ENE \\
\hline Jul-17 & 1.66 & WNW & 1.11 & NW & 5.00 & ENE \\
\hline Aug-17 & 2.50 & NNW & 1.39 & $\mathrm{NE}$ & 3.05 & NE \\
\hline Sep-17 & 2.22 & NNE & 2.22 & ENE & 3.89 & $\mathrm{NE}$ \\
\hline Oct-17 & 3.89 & SE & 4.17 & SSW & 3.89 & $\mathrm{NE}$ \\
\hline Nov-17 & 1.94 & NNE & 3.33 & SSW & 3.89 & ENE \\
\hline Dec-17 & 2.78 & SSE & 5.83 & SSW & 3.61 & $\mathrm{NE}$ \\
\hline Jan-18 & 3.61 & SSE & 9.73 & WSW & 3.61 & SW \\
\hline Feb-18 & 2.22 & ENE & 3.61 & SSW & 4.17 & SSW \\
\hline Mar-18 & 3.33 & NNE & 3.05 & SSW & 5.00 & SW \\
\hline Apr-18 & 3.05 & $\mathrm{~N}$ & 2.78 & WSW & 3.89 & SW \\
\hline May-18 & 1.39 & NNE & 2.78 & SW & 3.89 & SW \\
\hline
\end{tabular}

Note: The directions of wind N (North), E (East), S (South), and W (West).

A following wind rose represents the wind blowing frequency from specific directions over a particular stage for three study sites during the sampling period (Figure 4).
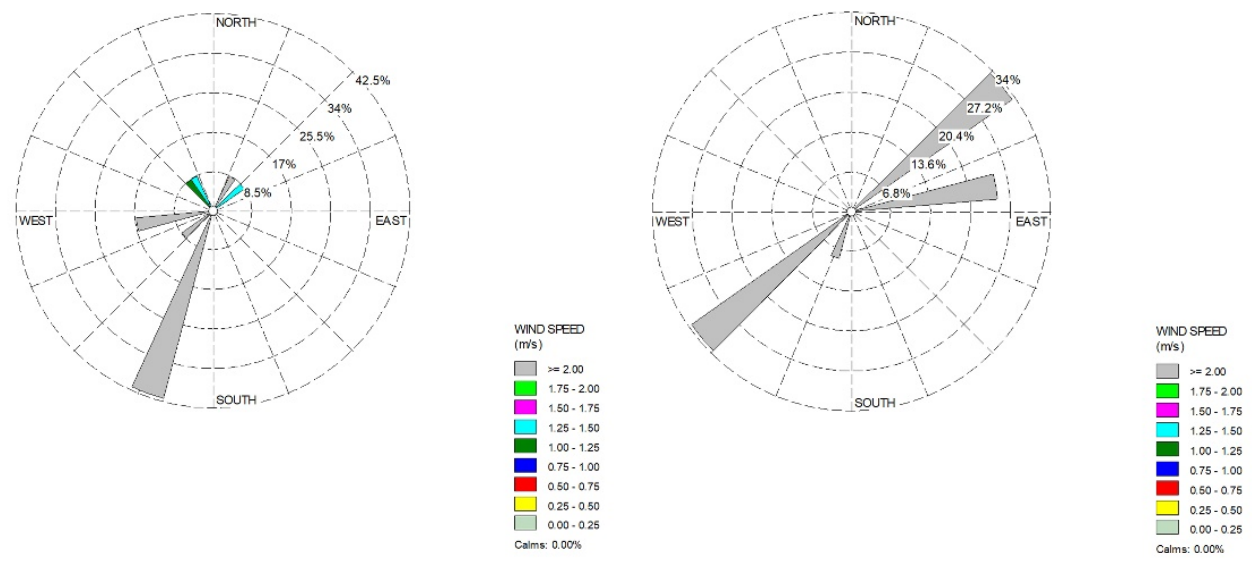

(a)

(b)

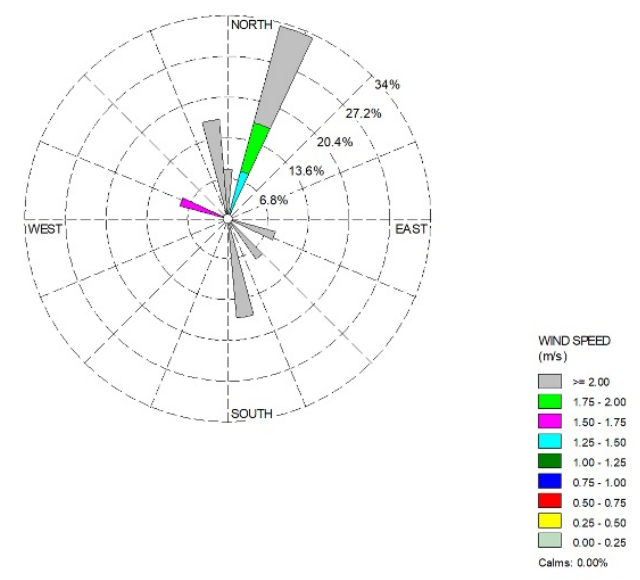

(c)

Figure 4. Wind trajectories of Karakoram (a), Himalaya (b), and (c) Hindukush. 
In the Karakoram, the meteorological conditions were quiet to calm, and at other times the wind velocity was usually greater than $2 \mathrm{~m} / \mathrm{s}$. As the annual wind rose shows (Figure 4a), most winds occurred southwards and southwestwards. Furthermore, between sampling duration, the wind was characterized by south-west to southwards directions with average speed remains more than $2 \mathrm{~m} / \mathrm{s}$.

In the Himalayas, meteorological conditions were also quiet, and at other times the wind velocity was also more than $2 \mathrm{~m} / \mathrm{s}$. As the annual wind rose shows (Figure 4b), most winds occurred southwestwards and northeastwards with an average speed remains above $2 \mathrm{~m} / \mathrm{s}$.

In Hindukush, overall meteorological conditions were low to quiet, and at other times the wind velocity was remained around $2 \mathrm{~m} / \mathrm{s}$ or lower. As the annual wind rose shows (Figure 4c), most winds occurred southwards, northeastwards, and northwards. Wind was dominated by the northward direction wind and average speed around $1.8 \mathrm{~m} / \mathrm{s}$ to $2 \mathrm{~m} / \mathrm{s}$ during sampling period.

\section{Results}

An inventory of different pollutants at three glaciers in Karakoram, Hindukush, and the Himalayas has been recorded for twelve months. The average concentration of ozone recorded in Hindukush, Karakoram, and Himalaya was $26.10 \pm 3.11,25.30 \pm 3.68$, and $33.03 \pm 3.94 \mu \mathrm{g} / \mathrm{m}^{3}$, respectively (Table 3). The average $\mathrm{CO}_{2}$ concentration $(243.03 \pm 31.19 \mathrm{ppm})$ was recorded at Himalaya followed by Hindukush and Karakoram with $191.99 \pm 24.64$ and $190.72 \pm 38.38$ ppm concentrations, respectively. Sulfur dioxide followed the same trend with average concentrations $2.01 \pm 0.31,1.59 \pm 0.25$, and $1.58 \pm 0.42$ ppm for Himalaya, Hindukush, and Karakoram, respectively. Moreover, the average concentration of nitrogen dioxide was recorded at Himalaya (3.30 \pm 0.35 ppm) followed by Karakoram (2.62 \pm 0.49 ppm) and Hindukush (2.60 \pm 0.27 ppm).

Table 3. Concentration of greenhouse gases at study site.

\begin{tabular}{|c|c|c|c|c|c|c|c|c|c|c|c|c|c|c|c|}
\hline Gases & Sites & Jun-17 & Jul-17 & $\begin{array}{c}\text { Aug- } \\
17\end{array}$ & $\begin{array}{c}\text { Sep- } \\
17\end{array}$ & Oct-17 & $\begin{array}{c}\text { Nov- } \\
17\end{array}$ & $\begin{array}{c}\text { Dec- } \\
17\end{array}$ & Jan-18 & $\begin{array}{c}\text { Feb- } \\
18\end{array}$ & $\begin{array}{c}\text { Mar- } \\
18\end{array}$ & $\begin{array}{c}\text { Apr- } \\
18\end{array}$ & $\begin{array}{c}\text { May- } \\
18\end{array}$ & Mean & SD \\
\hline \multirow{3}{*}{$\begin{array}{c}\mathrm{O}_{3} \\
\left(\mu \mathrm{g} / \mathrm{m}^{3}\right)\end{array}$} & Hindukush & 24.22 & 28.84 & 31.14 & 29.99 & 28.84 & 27.68 & 24.80 & 22.49 & 21.91 & 24.22 & 23.07 & 25.95 & 26.10 & 3.11 \\
\hline & Karakorum & 23.74 & 24.51 & 25.09 & 27.89 & 26.82 & 27.68 & 21.48 & 26.99 & 24.11 & 20.59 & 20.99 & 33.74 & 25.30 & 3.68 \\
\hline & Himalaya & 30.66 & 36.50 & 39.42 & 37.96 & 36.50 & 35.04 & 31.39 & 28.47 & 27.74 & 30.66 & 29.20 & 32.85 & 33.03 & 3.94 \\
\hline \multirow{3}{*}{$\begin{array}{l}\mathrm{CO}_{2} \\
\mathrm{ppm}\end{array}$} & Hindukush & 153.40 & 216.84 & 211.07 & 149.37 & 155.13 & 213.96 & 205.31 & 206.46 & 200.11 & 195.50 & 199.54 & 197.23 & 191.99 & 24.64 \\
\hline & Karakorum & 150.33 & 184.31 & 188.65 & 138.91 & 144.27 & 213.96 & 196.11 & 247.75 & 220.13 & 166.18 & 181.58 & 256.40 & 190.72 & 38.38 \\
\hline & Himalaya & 194.18 & 274.48 & 267.18 & 189.07 & 196.37 & 270.83 & 259.88 & 261.34 & 253.31 & 247.47 & 252.58 & 249.66 & 243.03 & 31.19 \\
\hline \multirow{3}{*}{$\begin{array}{l}\mathrm{SO}_{2} \\
\mathrm{ppm}\end{array}$} & Hindukush & 1.53 & 1.28 & 1.41 & 1.27 & 1.26 & 1.72 & 1.85 & 1.99 & 1.86 & 1.70 & 1.64 & 1.56 & 1.59 & 0.25 \\
\hline & Karakorum & 1.50 & 1.09 & 1.11 & 1.19 & 1.17 & 1.72 & 1.83 & 2.39 & 2.05 & 1.45 & 1.50 & 2.03 & 1.58 & 0.42 \\
\hline & Himalaya & 1.93 & 1.62 & 1.79 & 1.61 & 1.59 & 2.18 & 2.34 & 2.52 & 2.36 & 2.15 & 2.08 & 1.98 & 2.01 & 0.31 \\
\hline \multirow{3}{*}{$\begin{array}{l}\mathrm{NO}_{2} \\
\mathrm{ppm}\end{array}$} & Hindukush & 2.68 & 2.79 & 2.43 & 2.05 & 2.18 & 2.80 & 2.83 & 2.86 & 2.84 & 2.80 & 2.46 & 2.53 & 2.60 & 0.27 \\
\hline & Karakorum & 2.63 & 2.37 & 2.42 & 1.91 & 2.03 & 2.80 & 2.79 & 3.43 & 3.13 & 2.38 & 2.24 & 3.29 & 2.62 & 0.49 \\
\hline & Himalaya & 3.39 & 3.53 & 3.07 & 2.60 & 2.76 & 3.55 & 3.58 & 3.62 & 3.60 & 3.54 & 3.12 & 3.20 & 3.30 & 0.35 \\
\hline
\end{tabular}

The particulate matter (PM) concentrations were also recorded at the study sites. $\mathrm{PM}_{2.5}$ average concentration recorded at the Hindukush, Karakoram, and Himalayas was $14.75 \pm 2.91,14.28 \pm 3.34$, and $18.68 \pm 3.69 \mu \mathrm{g} / \mathrm{m}^{3}$, respectively. The average concentration of $\mathrm{PM}_{10}$ at Himalaya was $32.73 \pm 2.53$, followed by Hindukush $(25.86 \pm 2.00)$ and Karakoram (25.58 \pm 4.11$)$. Total suspended particles (TSP) followed the same trend with the highest concentration at the Himalayas $\left(88.93 \pm 10.20 \mu \mathrm{g} / \mathrm{m}^{3}\right)$. At the Hindukush and Karakoram, the average concentration was $70.25 \pm 8.05$ and $68.96 \pm 12.32 \mu \mathrm{g} / \mathrm{m}^{3}$, respectively (Table 4). 
Table 4. Concentration of particulate matter at study site.

\begin{tabular}{|c|c|c|c|c|c|c|c|c|c|c|c|c|c|c|c|}
\hline Pollutants & Site & $\begin{array}{c}\text { Jun- } \\
17\end{array}$ & Jul-17 & $\begin{array}{c}\text { Aug- } \\
17\end{array}$ & $\begin{array}{l}\text { Sep- } \\
17\end{array}$ & $\begin{array}{l}\text { Oct- } \\
17\end{array}$ & $\begin{array}{c}\text { Nov- } \\
17\end{array}$ & $\begin{array}{c}\text { Dec- } \\
17\end{array}$ & Jan-18 & $\begin{array}{c}\text { Feb- } \\
18\end{array}$ & $\begin{array}{c}\text { Mar- } \\
18\end{array}$ & $\begin{array}{c}\text { Apr- } \\
18\end{array}$ & $\begin{array}{c}\text { May- } \\
18\end{array}$ & Mean & SD \\
\hline \multirow{3}{*}{$\begin{array}{l}\mathrm{PM}_{2.5} \\
\left(\mu \mathrm{g} / \mathrm{m}^{3}\right)\end{array}$} & Hindukush & 15.57 & 17.88 & 13.84 & 13.84 & 12.69 & 15.57 & 20.18 & 19.03 & 12.11 & 11.53 & 12.69 & 12.11 & 14.75 & 2.91 \\
\hline & Karakorum & 15.26 & 15.20 & 15.55 & 12.87 & 11.80 & 15.57 & 11.87 & 22.84 & 13.32 & 9.80 & 11.55 & 15.74 & 14.28 & 3.34 \\
\hline & Himalaya & 19.71 & 22.63 & 17.52 & 17.52 & 16.06 & 19.71 & 25.55 & 24.09 & 15.33 & 14.60 & 16.06 & 15.33 & 18.68 & 3.69 \\
\hline \multirow{3}{*}{$\begin{array}{c}\mathrm{PM}_{10} \\
\left(\mu \mathrm{g} / \mathrm{m}^{3}\right)\end{array}$} & Hindukush & 23.64 & 28.84 & 25.95 & 24.22 & 23.64 & 28.26 & 28.26 & 28.26 & 24.80 & 24.22 & 25.37 & 24.80 & 25.86 & 2.00 \\
\hline & Karakorum & 23.17 & 24.51 & 25.09 & 22.53 & 21.99 & 28.26 & 24.30 & 33.91 & 27.28 & 20.59 & 23.09 & 32.24 & 25.58 & 4.11 \\
\hline & Himalaya & 29.93 & 36.50 & 32.85 & 30.66 & 29.93 & 35.77 & 35.77 & 35.77 & 31.39 & 30.66 & 32.12 & 31.39 & 32.73 & 2.53 \\
\hline \multirow{3}{*}{$\begin{array}{c}\text { TSP } \\
\left(\mu \mathrm{g} / \mathrm{m}^{3}\right)\end{array}$} & Hindukush & 67.84 & 80.81 & 68.84 & 65.85 & 62.85 & 75.82 & 83.81 & 81.81 & 63.85 & 61.86 & 65.85 & 63.85 & 70.25 & 8.05 \\
\hline & Karakorum & 66.49 & 68.69 & 70.31 & 61.24 & 58.45 & 75.82 & 62.58 & 98.17 & 70.24 & 52.58 & 59.92 & 83.01 & 68.96 & 12.32 \\
\hline & Himalaya & 85.88 & 102.29 & 87.14 & 83.35 & 79.56 & 95.98 & 106.08 & 103.56 & 80.83 & 78.30 & 83.35 & 80.83 & 88.93 & 10.20 \\
\hline
\end{tabular}

Apart from air, black carbon concentrations were also recorded in river water, glacier and snow $/$ rain. The mean BC value at Himalaya in river water was $\left(104 \pm 24.40 \mu \mathrm{g} / \mathrm{m}^{3}\right)$, glacier $\left(21.80 \pm 0.78 \mu \mathrm{g} / \mathrm{m}^{3}\right)$ and snow $/ \mathrm{rain}(67.84 \pm 24.40 \mathrm{ng} / \mathrm{g})$. At Hindukush, the concentrations were $82.43 \pm 19.27,15.50 \pm 0.71\left(\mu \mathrm{g} / \mathrm{m}^{3}\right)$, and $53.60 \pm 19.27 \mathrm{ng} / \mathrm{g}$, respectively. The average concentrations recorded at Karakoram were $79.44 \pm 13.79,15.69 \pm 0.96\left(\mu \mathrm{g} / \mathrm{m}^{3}\right)$ and $50.84 \pm 15.31 \mathrm{ng} / \mathrm{g}$, respectively (Table 5).

Table 5. Concentration of black carbon at study site.

\begin{tabular}{|c|c|c|c|c|c|c|c|c|c|c|c|c|c|c|c|}
\hline Black Carbon & Site & Jun-17 & Jul-17 & Aug-17 & Sep-17 & Oct-17 & Nov-17 & Dec-17 & Jan-18 & Feb-18 & Mar-18 & Apr-18 & May-18 & Mean & SD \\
\hline BC River & Hindukush & 80.46 & 83.28 & 105.84 & 90.80 & 71.41 & 75.61 & 80.23 & 61.83 & 94.90 & 118.17 & 82.09 & 44.58 & 82.43 & 19.27 \\
\hline Water & Karakorum & 78.85 & 70.78 & 72.45 & 84.45 & 66.41 & 75.61 & 93.00 & 74.19 & 104.39 & 100.45 & 74.70 & 57.95 & 79.44 & 13.79 \\
\hline$\mu \mathrm{g} / \mathrm{m}^{3}$ & Himalaya & 101.84 & 105.41 & 133.98 & 114.94 & 90.39 & 95.72 & 101.55 & 78.26 & 120.13 & 149.58 & 103.91 & 56.43 & 104.34 & 24.40 \\
\hline & Hindukush & 14.61 & 14.46 & 14.69 & 14.82 & 15.32 & 15.75 & 15.90 & 15.97 & 16.53 & 16.17 & 16.24 & 15.58 & 15.50 & 0.71 \\
\hline BC Glacier & Karakorum & 14.32 & 14.81 & 15.16 & 16.21 & 15.37 & 17.44 & 16.39 & 15.62 & 16.35 & 16.72 & 15.44 & 14.41 & 15.69 & 0.96 \\
\hline$\mu \mathrm{g} / \mathrm{m}^{3}$ & $\begin{array}{l}\text { Harakoya } \\
\text { Himalaya }\end{array}$ & $\begin{array}{l}14.02 \\
21.41\end{array}$ & $\begin{array}{l}14.01 \\
22.52\end{array}$ & $\begin{array}{l}15.10 \\
21.56\end{array}$ & $\begin{array}{l}10.21 \\
22.92\end{array}$ & 20.48 & $\begin{array}{l}17.44 \\
21.29\end{array}$ & $\begin{array}{l}1.39 \\
22.83\end{array}$ & $\begin{array}{l}22.41 \\
22.41\end{array}$ & 22.11 & $\begin{array}{l}11.72 \\
21.89\end{array}$ & $\begin{array}{l}11.44 \\
21.34\end{array}$ & $\begin{array}{l}14.41 \\
20.82\end{array}$ & $\begin{array}{l}15.09 \\
21.80\end{array}$ & 0.78 \\
\hline BC & Hindukush & 51.62 & 54.44 & 77.01 & 61.97 & 42.57 & 46.78 & 51.39 & 32.99 & 66.07 & 89.34 & 53.25 & 15.74 & 53.60 & 19.27 \\
\hline Snow/Rain & Karakorum & 50.59 & 46.27 & 47.36 & 57.63 & 39.59 & 46.78 & 64.74 & 39.59 & 72.67 & 75.94 & 48.46 & 20.46 & 50.84 & 15.31 \\
\hline$(\mathrm{ng} / \mathrm{g})$ mass & Himalaya & 65.34 & 68.91 & 97.48 & 78.44 & 53.89 & 59.22 & 65.05 & 41.76 & 83.63 & 113.08 & 67.41 & 19.93 & 67.84 & 24.40 \\
\hline
\end{tabular}

\subsection{Black Carbon and White-Sky Albedo}

According to the results presented in Table 6, the average BC concentrations with standard deviation were $2.35 \pm 0.94,3.30 \pm 1.35$, and $2.75 \pm 1.29\left(\mu \mathrm{g} / \mathrm{m}^{3}\right)$ for Karakoram, Himalaya, and Hindukush, respectively. Moreover, as BC data was observed monthly from June 2017 to May 2018, the statistical (annual) range of BC values were 3.19, 4.52, and 4.11 which is greater than their respective averages. However, variance remained quite low for the $\mathrm{BC}$ concentrations of Karakoram, Himalaya, and Hindukush.

Table 6. Concentration of black carbon, WSA, and cloud cover at the study site.

\begin{tabular}{|c|c|c|c|c|c|c|c|c|c|}
\hline \multirow[b]{2}{*}{ Month } & \multicolumn{3}{|c|}{$\begin{array}{l}\text { Hindukush } \\
\text { (Meragram) }\end{array}$} & \multicolumn{3}{|c|}{$\begin{array}{l}\text { Karakoram } \\
\text { (Passu) }\end{array}$} & \multicolumn{3}{|c|}{$\begin{array}{c}\text { Himalaya } \\
\text { (Raikot) }\end{array}$} \\
\hline & BC $\mu \mathrm{g} / \mathrm{m}^{3}$ & WSA & $\begin{array}{c}\text { Cloud } \\
\text { Cover } \\
(\%)\end{array}$ & BC $\mu \mathrm{g} / \mathrm{m}^{3}$ & WSA * & $\begin{array}{c}\text { Cloud } \\
\text { Cover } \\
(\%)\end{array}$ & ВС $\mu \mathrm{g} / \mathrm{m}^{3}$ & WSA & $\begin{array}{c}\text { Cloud } \\
\text { Cover } \\
(\%)\end{array}$ \\
\hline Jun-17 & 3.32 & 0.048 & 2 & 2.35 & 0.086 & 11 & 0.054 & 0.044 & 11 \\
\hline Jul-17 & 3.18 & 0.050 & 4 & 1.84 & 0.099 & 18 & 0.067 & 0.050 & 18 \\
\hline Aug-17 & 2.32 & 0.054 & 5 & 1.55 & 0.085 & 32 & 0.067 & 0.051 & 4 \\
\hline Sep-17 & 1.85 & 0.029 & 5 & 0.99 & 0.038 & 61 & 0.037 & 0.030 & 2 \\
\hline Oct-17 & 1.29 & 0.023 & 5 & 1.10 & 0.032 & 6 & 0.028 & 0.022 & 0 \\
\hline Nov-17 & 1.04 & 0.025 & 50 & 2.71 & 0.028 & 29 & 0.029 & 0.023 & 30 \\
\hline Dec-17 & 1.77 & 0.024 & 43 & 2.14 & 0.023 & 11 & 0.027 & 0.052 & 49 \\
\hline Jan-18 & 2.31 & 0.069 & 47 & 2.18 & 0.039 & 10 & 0.039 & 0.064 & 17 \\
\hline Feb-18 & 2.34 & 0.054 & 47 & 2.53 & 0.052 & 4 & 0.066 & 0.061 & 61 \\
\hline Mar-18 & 3.76 & 0.081 & 32 & 3.14 & 0.050 & 6 & 0.055 & 0.071 & 59 \\
\hline Apr-18 & 4.71 & 0.058 & 3 & 3.53 & 0.050 & 2 & 0.035 & 0.054 & 2 \\
\hline May-18 & 5.15 & 0.032 & 17 & 4.18 & 0.059 & 43 & 0.037 & 0.034 & 29 \\
\hline
\end{tabular}


Table 6. Cont.

\begin{tabular}{|c|c|c|c|c|c|c|c|c|c|}
\hline \multirow[b]{2}{*}{ Month } & \multicolumn{3}{|c|}{$\begin{array}{l}\text { Hindukush } \\
\text { (Meragram) }\end{array}$} & \multicolumn{3}{|c|}{$\begin{array}{c}\text { Karakoram } \\
\text { (Passu) }\end{array}$} & \multicolumn{3}{|c|}{$\begin{array}{c}\text { Himalaya } \\
\text { (Raikot) }\end{array}$} \\
\hline & BC $\mu \mathrm{g} / \mathrm{m}^{3}$ & WSA & $\begin{array}{c}\text { Cloud } \\
\text { Cover } \\
(\%)\end{array}$ & BC $\mu \mathrm{g} / \mathrm{m}^{3}$ & WSA * & $\begin{array}{c}\text { Cloud } \\
\text { Cover } \\
(\%)\end{array}$ & BC $\mu \mathrm{g} / \mathrm{m}^{3}$ & WSA & $\begin{array}{c}\text { Cloud } \\
\text { Cover } \\
(\%)\end{array}$ \\
\hline Mean & 2.752 & 0.0456 & 21.667 & 2.3531 & 0.0534 & 19.417 & 3.3018 & 0.0451 & 23.500 \\
\hline Range & 4.109 & 0.040 & 48.000 & 3.1857 & 0.076 & 59.000 & 4.5196 & 0.058 & 61.000 \\
\hline S.D & 1.296 & 0.019 & 20.340 & 0.9483 & 0.0246 & 18.263 & 1.3527 & 0.0157 & 22.310 \\
\hline Variance & 1.681 & 0.0002 & 413.697 & 0.8993 & 0.0006 & 333.538 & 1.8299 & 0.0004 & 497.727 \\
\hline Maximum & 5.146 & 0.081 & 50.000 & 4.1765 & 0.099 & 61.000 & 5.7474 & 0.067 & 61.000 \\
\hline Minimum & 1.037 & 0.0230 & 2.000 & 0.9908 & 0.0230 & 2.000 & 1.2279 & 0.0270 & 0.000 \\
\hline
\end{tabular}

* White-sky albedo; S.D. Standard deviation.

Similarly, Table 6 also summarizes the satellite-based WSA yielding WSA average values of sampling time (same as BC) with a standard deviation of $0.0534 \pm 0.024,0.0451 \pm 0.015$, and $0.0456 \pm 0.019$ for the Karakoram, Himalayas, and Hindukush, respectively. Also, in the same period i.e., from June 2017 to May 2018, variance for WSA values remains low for Karakoram, Himalaya and Hindukush. Monthly periodic observed data for BC along with respective satellite-based white-sky albedo starting from June 2017 to May 2018 is given in Table 6.

After performing all the data processing steps following monthly interpolated WSA map has been developed to represent monthly change of WSA within the study area as shown in Figure 5.

To temporally map the monthly values of BC and WSA, the following graphs have been generated to understand the temporal trend of BC and WSA as shown in Figure 5. As it is obvious from Figure 5 that $\mathrm{BC}$ for all three ranges remains intact while WSA shows relatively greater dispersion as compared with BC for Karakoram, Himalaya, and Hindukush. It also results that with increasing BC, WSA values decrease. However, there is no existing empirical relationship exists between BC and WSA. It is also obvious from descriptive statistics of the data (Table 6) that variance among WSA is greater than the variance in the $B C$.

To establish a relationship and to perform numerical analysis for WSA against the BC values, statistical technique of regression has been performed. It is to be noted that BC is considered as an independent variable while WSA is considered as dependent variable. To determine the second-degree polynomial regression curves for Karakoram, Himalaya, and Hindukush, respective values are given in Table 6. Plotting the above data, following graphs have been produced to understand the non-empirical relationship between BC and WSA for Karakoram, Himalaya and Hindukush. It is to be noted that to understand the BC-WSA relationship, the second-degree quadratic equation has been computed of BC values for all three ranges. Initial conditions in the snowpack with different BC concentrations are very similar, leading to almost indistinguishable WSA values. Hindukush showed a relatively more decreasing trend (Figure 6a) of WSA values with increasing BC, Karakoram range shows a slightly decreasing trend (Figure 6b). However, Himalaya (Figure 6c) shows an increasing trend of $\mathrm{BC}$. Nevertheless, the overall trend is a decreasing trend with increasing BC (Figure 6d). 

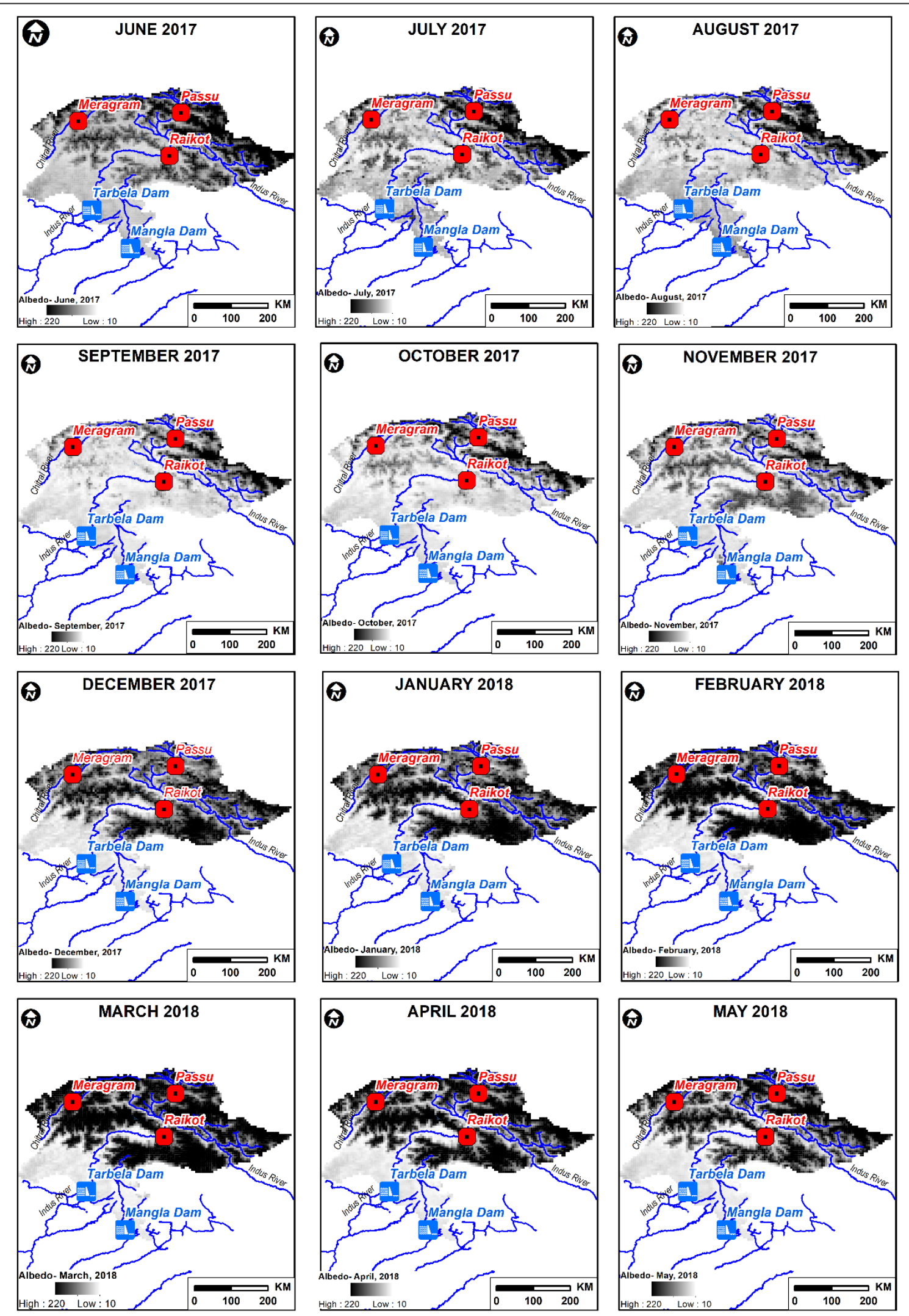

Figure 5. Monthly average of white-sky albedo. 


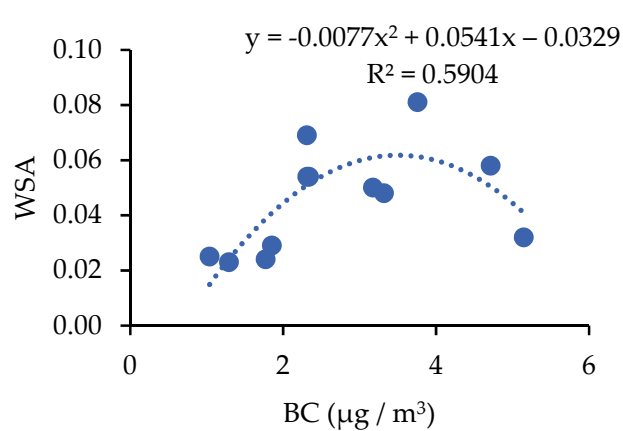

(a)

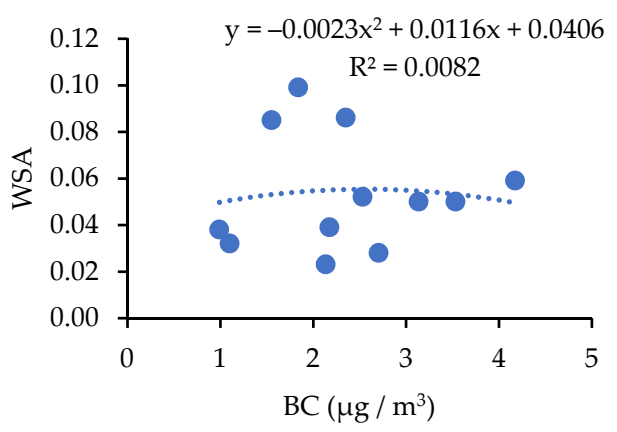

(c)

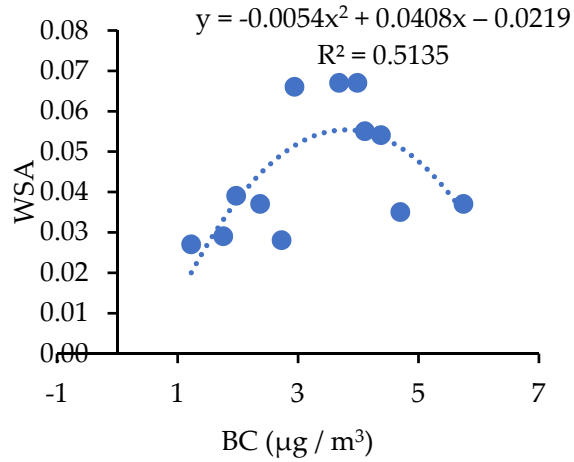

(b)

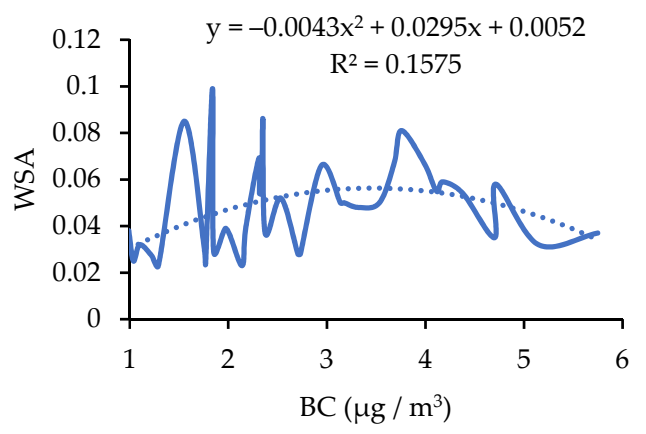

(d)

Figure 6. Trend resulting from regression model of BC and WSA (a) Hindukush, (b) Karakoram, (c) Himalaya, (d) cumulative graph of three mountain ranges.

\subsection{Wind Trajectories}

BC concentrations were measured for a year (June 2017-May 2018) to investigate (1) the diurnal heterogeneity of $\mathrm{BC}$ aerosols; (2) the relative dominance of biomass and fossil fuel sources resulting in $\mathrm{BC}$ heterogeneity; (3) the role of long-range and mountain wind transport, and overall meteorological influences on $\mathrm{BC}$ pollution; and (4) to compare BC concentrations over the HKH region in Pakistan [29]. Since fine particulate matter is transported from low-lying areas by upslope mountain wind, the concentration of fine particulate matter is observed to be high [30]. Since BC aerosols are primarily restricted in this fine size area of aerosols, this up-slope transport of aerosol particles may be well associated with the up-slope transport of BC aerosols. Thus, upslope transport of BC aerosols by mountain wind is found to compensate for the study period's low anthropogenic activity [29]. During the sampling period, the wind blowing frequency from specific directions was observed as, in Hindukush, overall meteorological conditions were low to quiet, and at other times the wind velocity remained around $2 \mathrm{~m} / \mathrm{s}$ or lower, with most winds occurring southwards, northeastwards, and northwards. During the sampling period, the wind was dominated by a northerly breeze with an average speed of $1.8 \mathrm{~m} / \mathrm{s}$ to $2 \mathrm{~m} / \mathrm{s}$. The meteorological conditions in the Karakoram were calm to calm, and the wind velocity was frequently greater than $2 \mathrm{~m} / \mathrm{s}$. The majority of the winds were from the south and southwest. Furthermore, the wind was characterized by south-west to southwards directions with an average speed of more than $2 \mathrm{~m} / \mathrm{s}$ between sampling durations. Meteorological conditions in Himalaya were also calm, with wind speeds of more than $2 \mathrm{~m} / \mathrm{s}$ at times. Most of the winds were from the southwest and north-east, with the average speeds being over $2 \mathrm{~m} / \mathrm{s}$. 


\subsection{Box Whisker Plot}

Following Figure 7 shows box and whisker plot of BC showing inter-quartile range and other statistical parameters (as described in Table 6). According to Figure 7a, the variation between $B C$ values was maximum in summer and minimum in summer while in the case of Aledo, variation in values were maximum in winter and minimum in spring as shown in Figure $7 \mathrm{~b}$.

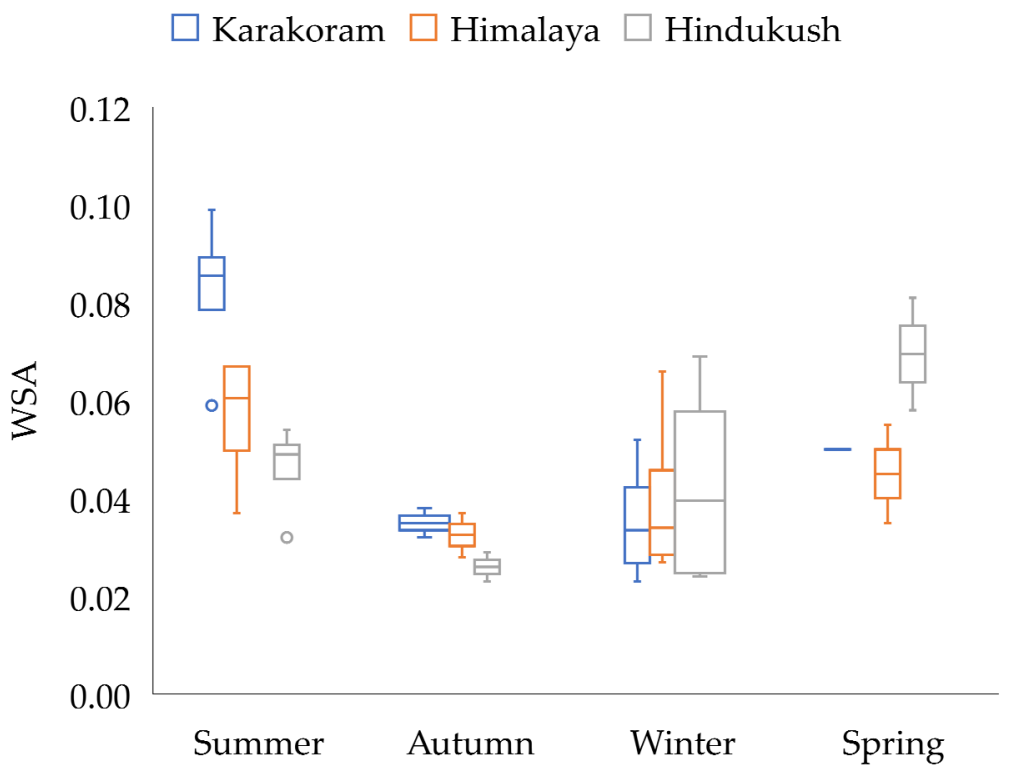

(a)

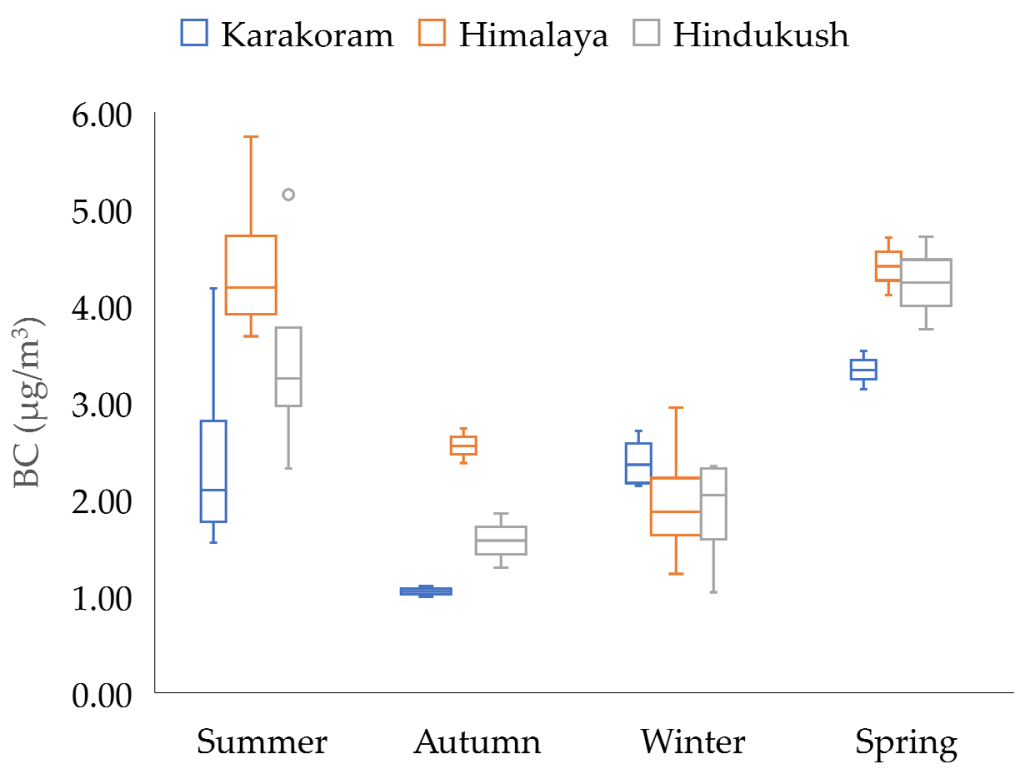

(b)

Figure 7. Box whisker analysis for WSA (a) and (b) BC.

\section{Discussion}

\subsection{Effects of Climatic Factors}

The HKH is vulnerable to climate change. Air pollutants generated inside and near the HKH magnify the impacts of greenhouse gases and accelerate cryosphere melting via black carbon and dust deposition, monsoon circulation, and rainfall distribution across Asia. There is mounting evidence that a diverse array of airborne pollutants is considerably 
contributing to glacial retreat while wreaking havoc on the environment's health, ecosystem, and species. The findings provide a baseline black carbon and associated pollutants concentration that can have a negative impact on climate and air quality and are also 'temporarily present' in the atmosphere.

From 1951 to 2014 , the air over $\mathrm{HKH}$ warmed at a pace of $0.2{ }^{\circ} \mathrm{C}$ per decade, with a rate of $0.5^{\circ} \mathrm{C}$ per decade at elevations above $4000 \mathrm{~m}$ [31]. With the exception of the high-elevation Karakoram Himalayas, several locations have seen decreased snowfall and retreating glaciers during the last half-century, according to the report $[3,32]$.

The total amount of precipitation that falls each year is unlikely to change, but climate change and dust will shorten the melting period, which typically lasts well into the summer, to a few brief spring months. Capturing all of that water in the spring will be difficult for water management, potentially leading to shortages and turmoil for species to survive.

\subsection{Particulate Matter}

PM 10 and PM 2.5 were not in higher concentration at HKH just a little variation in comparison at three sites. Every year, an estimated 5 billion tonnes of desert dust enter the Earth's atmosphere. Some of it makes its way to the world's roof, the HKH, where it warms glaciers and speeds up runoff [33]. During the spring and summer, long-distance transportation of dust particles in elevated aerosol layers is a recurring phenomenon over the Indian subcontinent. Elevated aerosol layers transfer significant volumes of dust to the snow-covered slopes of high-mountain Asia during the snow accumulation season [34]. Furthermore, above $4000 \mathrm{~m}$, the influence of dust on snow darkening is larger than that of black carbon. Few data imply that dust plays a discernible role in the observed geographical heterogeneity of snowmelt and snowline trends over $\mathrm{HKH}$, and they highlight an increasing contribution of dust to snowmelt as the snowline rises with warming [35].

\subsection{Gases Emissions at $\mathrm{HKH}$ Glaciers $\left(\mathrm{CO}_{2}, \mathrm{NO}_{2}, \mathrm{SO}_{2}, \mathrm{O}_{3}\right)$}

The HKH glaciers are melting and shrinking due to global warming caused by increased anthropogenic greenhouse gas emissions. The current concentrations for $\mathrm{O}_{3}, \mathrm{CO}_{2}$, $\mathrm{SO}_{2}, \mathrm{NO}_{2}$ were $28.14 \pm 3.58,208.58 \pm 31.40,1.73 \pm 0.33,2.84 \pm 0.37$, respectively, and the future scenario could increase current concentrations with the high population density near these glaciers, deforestation, and land-use changes [36]. Many HKH glaciers have retreated by $7.3 \mathrm{~m}$ year from 1842 to 1935, and by almost 23\% annually in the following four decades [37]. The present glacier melt trends predict that the many perennial rivers that crisscross the Pakistan plain may soon become seasonal due to climate change, affecting the region's economies. Many Asian glaciers, such as those under $4 \mathrm{~km}$ in length in the HKH and Tibetan Plateau, are expected to vanish or shrink by more than $60 \%$ [38]. Rai and Gurung [39] has also reported that if the Earth continues to warm at the current rate, glaciers in the Himalaya will likely vanish by 2035 , if not sooner. Its current $500,000 \mathrm{~km}^{2}$ will likely fall to $100,000 \mathrm{~km}^{2}$ by 2035 . The Intergovernmental Panel on Climate Change (IPCC) report [31] also predicted same that by 2100, the HKH glaciers would have lost between a third and half of their mass. Scientists warn in the literature that $\mathrm{HKH}$ regions is warming five to six times quicker than the rest of the world and is also impacting Asian monsoons.

The Integrated Assessment of Black Carbon and Tropospheric Ozone was published in 2011 by the UNEP and the World Meteorological Organization (WMO), and was the first attempt to look at the potential to slow global warming by reducing particle pollution (black carbon) and ozone precursors. These pollutants, which were previously thought to be just conventional air pollutants, have a short-term impact on climate change [9,40].

\subsection{Black Carbon}

The in-situ observations of BC concentrations in precipitation, river water, and in the air from the HKH over three glaciers were the focus of this research. Results demonstrate that average $\mathrm{BC}$ concentrations in the air were lower than those reported for Indian and Nepalese sites [31]. In the 2017-2018 seasons, BC in the air from the glaciers at HKH 
was summarized in Figure 7. The highest BC levels were observed in summer (May) and the results were consistent with Kostrykin et al. [41], while the lowest was recorded in September. It was consistently low from February to May. According to Ming et al. [42], BC concentrations can be boosted by closeness to sources as well as melting, which lowers WSA. Due to variances in $\mathrm{BC}$ emissions or depositions, $\mathrm{BC}$ concentrations in the southeastern $\mathrm{HKH}$ glaciers were almost equivalent to those in the Tibet Plateau (TP) but were lower than those in Tien Shan and the northern TP [43,44]. In general, BC values in the high mountains of Asia are significantly greater than as compared with northern Greenland surface snow [45], alpine [46], and arctic [47] ice cores.

The amount of black carbon in the atmosphere varies throughout the year, directly altering the absorbance of solar radiation [48]. At Passu (Karakoram), BC concentrations were calculated as $2.39 \mu \mathrm{g} / \mathrm{m}^{3}$ in winter, $3.34 \mu \mathrm{g} / \mathrm{m}^{3}$ in spring, $2.48 \mu \mathrm{g} / \mathrm{m}^{3}$ in summer, and $1.05 \mu \mathrm{g} / \mathrm{m}^{3}$ in autumn. At Raikot (Himalaya) it was $1.97 \mathrm{ug} / \mathrm{m}^{3}$ in winter, $4.41 \mu \mathrm{g} / \mathrm{m}^{3}$ in spring, $4.45 \mu \mathrm{g} / \mathrm{m}^{3}$ in summer, and $2.55 \mu \mathrm{g} / \mathrm{m}^{3}$ in autumn. At Meragram (Hindukush) $\mathrm{BC}$ values were $1.86 \mathrm{ug} / \mathrm{m}^{3}$ in winter, $4.24 \mu \mathrm{g} / \mathrm{m}^{3}$ in spring, $3.49 \mu \mathrm{g} / \mathrm{m}^{3}$ in summer, and $1.57 \mu \mathrm{g} / \mathrm{m}^{3}$ in autumn.

The WSA pattern at the HKH glaciers shows an increasing trend from November to January (mean albedo: 0.040), then stays relatively constant in spring (0.051) before rising again in June, July, and August (0.055) and declining in autumn (0.051).

Cloud cover can also influence how much solar radiation a planet absorbs and how much sunlight it receives on its surface. In general, more cloud cover correlates with higher albedo and reduced solar energy absorption, as seen in the summer months, although BC concentration was rather high. Cloud cover has a significant impact on the Earth's energy budget, accounting for around half of total albedo [49,50]. Although WSA has a slightly inverse association with BC concentrations at $\mathrm{HKH}$ (Figure 6d), higher WSA was attributed to a larger proportion of cloud cover presence on sampling dates throughout the summer months. These BC concentrations in the air were later deposited on glacier surfaces (either on ice or snow). As the $\mathrm{BC}$ concentration rises, it absorbs more sunlight and diminishes WSA, causing the surface of glaciers to warm.

The findings suggest that the amount of $\mathrm{BC}$ in the air at $\mathrm{HKH}$ glaciers is likely to be lofted and then deposited, darkening the glacier surfaces, as has been observed at TP and Himalayan glaciers in the vicinity $[8,51]$. The results of the numerous studies are not always directly comparable due to differences in analytical methodology, sampling dates, and snow conditions. Nonetheless, these studies show a significant variety of BC concentrations across glaciers and localities. Similarly, many other studies such as Gertler et al. [52]; Kaspari et al. [49]; Ming et al. [53] observed that the average snow albedo loss induced by BC at glaciers ranged between 0.27 and 23 percent for the combined effect and were equivalent but recognized as low relevance to diminish the albedo at glaciers.

The amount of black carbon particles in the air varies with precipitation (rain and snow), which is subsequently deposited in ice, reducing snow albedo and influencing melting. Soot produced by incomplete combustion of fossil fuels, biofuels, and biomass is known as black carbon. Burning biofuels produces roughly $20 \%$, fossil fuels produce $38 \%$, and open biomass burning in forests and savannah produces $42 \%$ black carbon [54]. A variety of human activities, including industries, cars, biomass burning, forest fires, brick-making, and cook stoves, contribute to the pollution. When $\mathrm{BC}$ is released into the atmosphere as a result of these operations, it has the potential to travel vast distances (sometimes into the mountains) and settle on top of glaciers and snow. Once there, the BC lowers the snow's light- and heat-reflective capability, causing it to melt when the temperature rises due to the absorbed heat energy. The melting of snow and glaciers is accelerated as a result. BC deposition is responsible for up to $50 \%$ of the global increase in glacier and snow melt [48].

To address these glaciological concerns, it is necessary to have a good understanding of the feedbacks between climatic forcing and glacier reactions [17]. As a result, substantial data on glacier distribution, volume, mass-balance gradations, and landscape elements 
impacting ablation are necessary. In the examined area, the connection between black carbon concentration and WSA was anticipated to be inverse, with an increase in existing BC increasing glacier melt. Many studies have shown that $\mathrm{BC}$ concentrations can reduce albedo, which can accelerate snow /ice melt and trigger albedo feedback [9,44,49,51,52,55-58]. In recent years, $\mathrm{BC}$ has been identified as one of the most important forcing agents driving regional climate change and general air circulation in recent years, measuring this effect is difficult due to uncertainty [52,59-61].

Black carbon particles are mostly derived from fossil fuels and biomass [9]. Black carbon can warm the surroundings due to its capacity to absorb sun light at certain wavelengths. Some estimates suggest that BC has a significant impact on climate, such as reductions in precipitation and positive shortwave radiative forcing in the atmosphere, as the second-strongest climate warming forcing agent after carbon dioxide [8,9,60-62]. Other BC particles could have large-scale environmental implications, such as melting of glaciers in the Himalayas and elsewhere $[49,51,57,63]$. It is likely that even a small amount of BC deposited on a glacier's surface, whether by dry or wet processes, could reduce radiative forcing efficiency and cause glacial melt via lowering WSA.

The Hindukush, Himalayan, and Karakoram glaciers are melting at a pace of $0.3 \mathrm{~m}$ per year in their western parts. In the east, the retreat is three times faster than in the west [49]. According to one study, glaciers around Everest may shrink by $39 \%$ to $52 \%$ by 2050 . Even if we halted global warming immediately, we would lose $20 \%$ of Asia's glaciers [2]. Rapid glaciers and snow melt will cause natural disasters and threaten livelihoods in the mountains and downstream. Changes in hydrology, timing, and water quantity directly affect natural environments. This transformation has increased the vulnerability of wild species and communities in the region. Rapid glacier and snow melt has caused natural disasters and ecological disturbances in the mountains and downstream. The high level of biomass usage and increasing energy demands from coal-fired power plants in South Asia are increasing the quantity of $\mathrm{BC}$ circulating through the $\mathrm{HKH}$ mountain ranges and threatening to hasten glacier melt $[5,19,48,52]$.

Several studies, including those by Gertler et al. [52] and Yasunari et al. [64] have found that rapid glacier melt may result in an increase in yearly discharge of 11.6 to 33.9 percent if white-sky albedo reduces by 2.0 to 5.2 percent as a result of $\mathrm{BC}$ depositions in the HKH region. This process would be accelerated further if melting exposed additional pollutants at the glacier surface or if the melt season were extended as a result of global warming or macroscale air circulations $[43,65]$. After examining the present BC and WSA concentrations at HKH Pakistan, we can conclude that if the BC content is doubled in future scenarios as a result of increased traffic and industrial growth along with the road infrastructure, glacier melting can accelerate dramatically. Additionally, some earlier BC research revealed that microorganisms, such as the pigmented algae that live in snow and ice, can significantly reduce reflectivity $[46,66-68]$. The relative contribution of biological activity to the darkening of glacial surfaces against inorganic dust and $\mathrm{BC}$ remains an unanswered subject [66]. More research on black carbon emissions from biomass burning is certainly needed, especially in light of increased automobile pollution as a result of road infrastructure, human settlements, and industrial expansion.

\section{Conclusions}

Increasingly, scientists are concerned about environmental pollutants' behavior and their impacts on the cryosphere. This study examined white sky albedo, air contaminants, and black carbon concentrations. Aeroqual 500 and TSI DRX 8533 have been used to record a variety of contaminants at three glaciers in the Karakoram, Hindukush, and Himalaya regions. Black carbon was estimated using filter sampling and analyzed by DRI Model 2015, a multi-wavelength thermal/optical carbon analyzer, and satellite-based white-sky albedo (WSA) was downloaded from MODIS. The pollutant concentration at HKH was as follows: ozone $\left(28.14 \pm 3.58 \mu \mathrm{g} / \mathrm{m}^{3}\right)$, carbon dioxide $\left(208.58 \pm 31.40 \mu \mathrm{g} / \mathrm{m}^{3}\right)$, sulfur dioxide $\left(1.73 \pm 0.33 \mu \mathrm{g} / \mathrm{m}^{3}\right)$, nitrogen dioxide $\left(2.84 \pm 0.37 \mu \mathrm{g} / \mathrm{m}^{3}\right)$, PM2.5 $\left(15.90 \pm 3.32 \mu \mathrm{g} / \mathrm{m}^{3}\right)$, 
PM10 $\left(28.05 \pm 2.88 \mu \mathrm{g} / \mathrm{m}^{3}\right)$, total suspended particles $\left(76.05 \pm 10.19 \mu \mathrm{g} / \mathrm{m}^{3}\right)$, black carbon in river water $\left(88.74 \pm 19.16 \mu \mathrm{g} / \mathrm{m}^{3}\right)$, glaciers $\left(17.66 \pm 0.82 \mu \mathrm{g} / \mathrm{m}^{3}\right)$, snow $/$ rain $(57.43 \pm 19.66 \mathrm{ng} / \mathrm{g})$, and air $\left(2.80 \pm 1.20 \mu \mathrm{g} / \mathrm{m}^{3}\right)$. There were $2.35 \pm 0.94 \mu \mathrm{g} / \mathrm{m}^{3}$, $4.38 \pm 1.35 \mu \mathrm{g} / \mathrm{m}^{3}$, and $3.32 \pm 1.09\left(\mu \mathrm{g} / \mathrm{m}^{3}\right)$ of average BC concentrations in Karakoram, the Himalayas, and Hindukush, whereas the satellite-based WSA yields on sampling date (the same as BC) were $0.053 \pm 0.024,0.045 \pm 0.015$, and $0.045 \pm 0.019$. The link between WSA and BC was studied using a regression analysis tool. A clearer comprehension of the non-empirical connection between the measured BC and satellite-based WSA is provided by the derived curves. The research findings demonstrated that exogenous pollution has the potential to significantly impact the climatic and environmental conditions in $\mathrm{HKH}$. The findings of this study will be valuable for future research into the interactions of the atmosphere and cryosphere around the world. This research will help Pakistan's HKH region by providing data and a foundation for future research. In addition to addressing the key scientific problems raised, this study recommends several future research directions.

Author Contributions: Conceptualization, I.Z. and Z.A.; methodology, I.Z. and Z.A.; software, U.A. and S.T.R.; validation, Z.A. and R.A.; formal analysis, I.Z. and Z.Z.; investigation, I.Z., Z.A., U.A., R.A., S.S. and Z.Z.; resources, I.Z. and Z.A.; data curation, Z.A. and U.A.; writing-original draft preparation, I.Z. and Z.A. writing-review and editing, R.A., U.A., S.S., Z.Z. and S.T.R.; visualization, U.A. and S.T.R.; supervision, Z.A. All authors have read and agreed to the published version of the manuscript.

Funding: This research received no external funding.

Institutional Review Board Statement: Not applicable.

Informed Consent Statement: Not applicable.

Data Availability Statement: Not applicable.

Acknowledgments: We would like to acknowledge Chen Peng Fei for black carbon sample analysis from State Key Laboratory of Cryosphere Science, Northwest, Institute of Eco-Environment and Resources, Chinese Academy of Sciences, Lanzhou 730000, China. We are thankful for joint cooperation between University of the Punjab, Lahore, Pakistan; Yunnan University, Kunming, China; and China Postdoctoral System. Pir Shaukat Ali and Fatima Jabeen (ICIMOD) for advice and guidance. Furthermore, we are thankful to Mehboob (local) for field support and logistics. Zulqarnain Haider and Aliza Batool for preparation of infographics.

Conflicts of Interest: The authors declare no conflict of interest.

\section{References}

1. Marzeion, B.; Cogley, J.G.; Richter, K.; Parkes, D. Attribution of global glacier mass loss to anthropogenic and natural causes. Science 2014, 345, 919-921. [CrossRef] [PubMed]

2. Bolch, T.; Kulkarni, A.; Kääb, A.; Huggel, C.; Paul, F.; Cogley, J.G.; Frey, H.; Kargel, J.S.; Fujita, K.; Scheel, M. The state and fate of Himalayan glaciers. Science 2012, 336, 310-314. [CrossRef]

3. Brown, M.; Racoviteanu, A.; Tarboton, D.G.; Gupta, A.S.; Nigro, J.; Policelli, F.; Habib, S.; Tokay, M.; Shrestha, M.; Bajracharya, S. An integrated modeling system for estimating glacier and snow melt driven streamflow from remote sensing and earth system data products in the Himalayas. J. Hydrol. 2014, 519, 1859-1869. [CrossRef]

4. Gurung, D.R.; Giriraj, A.; Aung, K.S.; Shrestha, B.R.; Kulkarni, A.V. Snow-Cover Mapping and Monitoring in the Hindu KushHimalayas; International Centre for Integrated Mountain Development (ICIMOD): Patan, Nepal, 2011.

5. IPCC Intergovernmental Panel on Climate Change. The Physical Science Basis: Contribution of Working Group I to the Fourth Assessment Report of the Intergovernmental Panel on Climate Change. Intergov. Panel Clim. Change 2007, $2007,996$.

6. Moore, F.C. Climate change and air pollution: Exploring the synergies and potential for mitigation in industrializing countries. Sustainability 2009, 1, 43-54. [CrossRef]

7. Jacob, D.J. Introduction to Atmospheric Chemistry; Princeton University Press: Princeton, NJ, USA, 1999.

8. Ramanathan, V.; Carmichael, G. Global and regional climate changes due to black carbon. Nat. Geosci. 2008, 1, 221-227. [CrossRef]

9. Bond, T.C.; Doherty, S.J.; Fahey, D.W.; Forster, P.M.; Berntsen, T.; DeAngelo, B.J.; Flanner, M.G.; Ghan, S.; Kärcher, B.; Koch, D. Bounding the role of black carbon in the climate system: A scientific assessment. J. Geophys. Res. Atmos. 2013, 118, 5380-5552. [CrossRef] 
10. Beres, N.D.; Lapuerta, M.; Cereceda-Balic, F.; Moosmüller, H. Snow surface albedo sensitivity to black carbon: Radiative transfer modelling. Atmosphere 2020, 11, 1077. [CrossRef]

11. Beniston, M. Mountain Environments in Changing Climates; Routledge: Oxfordshire, UK, 2002; Volume 59, pp. 5-31. [CrossRef]

12. Meier, M.F.; Dyurgerov, M.B. How Alaska affects the world. Science 2002, 297, 350-351. [CrossRef]

13. Haeberli, W.; Hoelzle, M.; Suter, S. Into the second century of worldwide glacier monitoring: Prospects and strategies. J. Hydrol. Reg. Stud. 1998, 56. Available online: https://wgms.ch/downloads/Haeberli_1998.pdf (accessed on 8 July 2020).

14. Nakawo, M.; Fujita, K.; Ageta, Y.; Shankar, K.; Pokhrel, A.P. Basic studies for assessing the impacts of the global warming on the Himalayan cryosphere, 1994-1996. Bull. Glaciol. Res. 1997, 15, 53-58.

15. Molnar, P.; England, P. Late Cenozoic uplift of mountain ranges and global climate change: Chicken or egg? Nature 1990, 346, 29-34. [CrossRef]

16. Bishop, M.P.; Shroder, J.F., Jr.; Bonk, R.; Olsenholler, J. Geomorphic change in high mountains: A western Himalayan perspective Glob. Planet. Change 2002, 32, 311-329. [CrossRef]

17. Dyurgerov, M.B.; Meier, M.F. Twentieth century climate change: Evidence from small glaciers. Proc. Natl. Acad. Sci. USA 2000, 97, 1406-1411. [CrossRef]

18. Rasul, G.; Chaudhry, Q.; Mahmood, A.; Hyder, K.; Dahe, Q. Glaciers and glacial lakes under changing climate in Pakistan. Pakisan J. Meteorol. 2011, 8, 15. Available online: http://www.climateinfo.pk/frontend/web/attachments/data-type/1_Glaciers\%20 and\%20Glacial\%20Lakes\%20under\%20Changing\%20Climate\%20in\%20Pakistan.pdf (accessed on 8 July 2020).

19. Gul, C.; Mahapatra, P.S.; Kang, S.; Singh, P.K.; Wu, X.; He, C.; Kumar, R.; Rai, M.; Xu, Y.; Puppala, S.P. Black carbon concentration in the central Himalayas: Impact on glacier melt and potential source contribution. Environ. Pollut. 2021, 275, 116544. [CrossRef] [PubMed]

20. Williams, R.S.; Ferrigno, J.G.; Manley, W.F. Glaciers of Asia. In US Geological Survey Professional Paper; USGS: Washington, DC, USA, 2010; p. 349.

21. Glaciers of Pakistan. Available online: http://www.angelfire.com/al/badela/Glaciers.html (accessed on 20 September 2021).

22. Craig, T. Pakistan has more glaciers than almost anywhere on Earth. But they are at risk. The Washington Post, 12 August 2016.

23. Cogley, G. No ice lost in the Karakoram. Nat. Geosci. 2012, 5, 305-306. [CrossRef]

24. Kääb, A.; Berthier, E.; Nuth, C.; Gardelle, J.; Arnaud, Y. Contrasting patterns of early twenty-first-century glacier mass change in the Himalayas. Nature 2012, 488, 495-498. [CrossRef]

25. Jilani, R.H.; Naseer, A.; Paras, S.; Sher, M. Monitoring of Mountain Glacial Variations in Northern Pakistan, from 1992 to 2009 Using Landsat and ALOS data. In Proceedings of the Symposium 4th PI Symposium of JAXA, kyoto, Japan, 15-17 November 2010.

26. Schmidt, S.; Nüsser, M. Fluctuations of Raikot Glacier during the past 70 years: A case study from the Nanga Parbat massif, northern Pakistan. J. Glaciol. 2009, 55, 949-959. [CrossRef]

27. Zhang, Y.; Kang, S.; Li, C.; Gao, T.; Cong, Z.; Sprenger, M.; Liu, Y.; Li, X.; Guo, J.; Sillanpää, M. Characteristics of black carbon in snow from Laohugou No. 12 glacier on the northern Tibetan Plateau. Sci. Total Environ. 2017, 607, 1237-1249. [CrossRef] [PubMed]

28. Worldweather. Available online: worldweatheronline.com (accessed on 31 October 2021). [CrossRef]

29. Sarkar, C.; Chatterjee, A.; Singh, A.K.; Ghosh, S.K.; Raha, S. Characterization of black carbon aerosols over Darjeeling-A high altitude Himalayan station in eastern India. Aerosol Air Qual. Res. 2015, 15, 465-478. [CrossRef]

30. Adak, A.; Chatterjee, A.; Singh, A.K.; Sarkar, C.; Ghosh, S.; Raha, S. Atmospheric fine mode particulates at eastern Himalaya India: Role of meteorology, long-range transport and local anthropogenic sources. Aerosol Air Qual. Res. 2014, 14, 440-450. [CrossRef]

31. Stocker, T. Climate Change 2013: The Physical Science Basis: Working Group I Contribution to the Fifth Assessment Report of the Intergovernmental Panel on Climate Change; Cambridge University Press: Cambridge, UK, 2014.

32. Yao, T.; Xue, Y.; Chen, D.; Chen, F.; Thompson, L.; Cui, P.; Koike, T.; Lau, W.K.-M.; Lettenmaier, D.; Mosbrugger, V. Recent third pole's rapid warming accompanies cryospheric melt and water cycle intensification and interactions between monsoon and environment: Multidisciplinary approach with observations, modeling, and analysis. Bull. Am. Meteorol. Soc. 2019, 100, 423-444. [CrossRef]

33. Ghatak, D.; Sinsky, E.; Miller, J. Role of Snow-Albedo Feedback in High Elevation Warming. In Proceedings of the AGU Fall Meeting Abstracts, San Francisco, CA, USA, 10 October 2013; p. A11A-0002.

34. Zaveri, R.A.; Easter, R.C.; Fast, J.D.; Peters, L.K. Model for simulating aerosol interactions and chemistry (MOSAIC). J. Geophys. Res. Atmos. 2008, 113, D13. [CrossRef]

35. Sarangi, C.; Qian, Y.; Rittger, K.; Leung, L.R.; Chand, D.; Bormann, K.J.; Painter, T.H. Dust dominates high-altitude snow darkening and melt over high-mountain Asia. Nat. Clim. Chang. 2020, 10, 1045-1051. [CrossRef]

36. Sun, H.; Liu, X.; Pan, Z. Direct Radiative Effects of Dust Aerosols Emitted from the Tibetan Plateau on the East Asian Summer Monsoon-A Regional Climate Model Simulation. Atmos. Chem. Phys. 2017, 17, 13731-13745. [CrossRef]

37. Hasnain, S.I. Himalayan glaciers meltdown: Impact on South Asian Rivers. Int. Assoc. Hydrol. Sci. Publ. 2002, $274,417-423$. [CrossRef]

38. Shen, Y.; Wang, G.; Wu, Q.; Liu, S. The impact of future climate change on ecology and environments in the Changjiang-Yellow Rivers source region. J. Glaciol. Geocryol. 2002, 24, 308-314. 
39. Rai, S.C.; Gurung, T. An Overview of Glaciers, Glacier Retreat, and Subsequent Impacts in Nepal, India and China; ETDEWEB: Washington, DC, USA, 2005.

40. Chand, D.; Anderson, T.; Wood, R.; Charlson, R.; Hu, Y.; Liu, Z.; Vaughan, M. Quantifying above-cloud aerosol using spaceborne lidar for improved understanding of cloudy-sky direct climate forcing. J. Geophys. Res. Atmos. 2008, 113, D13206. [CrossRef]

41. Kostrykin, S.; Revokatova, A.; Chernenkov, A.; Ginzburg, V.; Polumieva, P.; Zelenova, M. Black Carbon Emissions from the Siberian Fires 2019: Modelling of the Atmospheric Transport and Possible Impact on the Radiation Balance in the Arctic Region. Atmosphere 2021, 12, 814. [CrossRef]

42. Ming, J.; Xiao, C.; Cachier, H.; Qin, D.; Qin, X.; Li, Z.; Pu, J. Black Carbon (BC) in the snow of glaciers in west China and its potential effects on albedos. Atmos. Res. 2009, 92, 114-123. [CrossRef]

43. Yang, S.; Xu, B.; Cao, J.; Zender, C.S.; Wang, M. Climate effect of black carbon aerosol in a Tibetan Plateau glacier. Atmos. Environ. 2015, 111, 71-78. [CrossRef]

44. Ming, J.; Xiao, C.; Du, Z.; Yang, X. An overview of black carbon deposition in High Asia glaciers and its impacts on radiation balance. Adv. Water Resour. 2013, 55, 80-87. [CrossRef]

45. Ruppel, M.; Isaksson, E.; Ström, J.; Beaudon, E.; Svensson, J.; Pedersen, C.; Korhola, A. Increase in elemental carbon values between 1970 and 2004 observed in a 300-year ice core from Holtedahlfonna (Svalbard). Atmos. Chem. Phys. 2014, 14, 11447-11460. [CrossRef]

46. Aoki, T.; Matoba, S.; Yamaguchi, S.; Tanikawa, T.; Niwano, M.; Kuchiki, K.; Adachi, K.; Uetake, J.; Motoyama, H.; Hori, M. Light-absorbing snow impurity concentrations measured on Northwest Greenland ice sheet in 2011 and 2012. Bull. Glaciol. Res. 2014, 32, 21-31. [CrossRef]

47. Thevenon, F.; Anselmetti, F.S.; Bernasconi, S.M.; Schwikowski, M. Mineral dust and elemental black carbon records from an Alpine ice core (Colle Gnifetti glacier) over the last millennium. J. Geophys. Res. Atmos. 2009, 114, D17. [CrossRef]

48. Clark, D. Emission Factors for Black Carbon; Cundall Johnston \& Partners LLP: Newcastle, UK, 2013.

49. Kaspari, S.; Painter, T.H.; Gysel, M.; Skiles, S.; Schwikowski, M. Seasonal and elevational variations of black carbon and dust in snow and ice in the Solu-Khumbu, Nepal and estimated radiative forcings. Atmos. Chem. Phys. 2014, 14, 8089-8103. [CrossRef]

50. Qu, B.; Ming, J.; Kang, S.-C.; Zhang, G.-S.; Li, Y.-W.; Li, C.-D.; Zhao, S.-Y.; Ji, Z.-M.; Cao, J.-J. The decreasing albedo of the Zhadang glacier on western Nyainqentanglha and the role of light-absorbing impurities. Atmos. Chem. Phys. 2014, 14, 11117-11128. [CrossRef]

51. Xu, B.; Cao, J.; Hansen, J.; Yao, T.; Joswia, D.R.; Wang, N.; Wu, G.; Wang, M.; Zhao, H.; Yang, W. Black soot and the survival of Tibetan glaciers. Proc. Natl. Acad. Sci. USA 2009, 106, 22114-22118. [CrossRef] [PubMed]

52. Gertler, C.G.; Puppala, S.P.; Panday, A.; Stumm, D.; Shea, J. Black carbon and the Himalayan cryosphere: A review. Atmos. Environ. 2016, 125, 404-417. [CrossRef]

53. Ming, J.; Cachier, H.; Xiao, C.; Qin, D.; Kang, S.; Hou, S.; Xu, J. Black carbon record based on a shallow Himalayan ice core and its climatic implications. Atmos. Chem. Phys. 2008, 8, 1343-1352. [CrossRef]

54. Bond, T.C.; Streets, D.G.; Yarber, K.F.; Nelson, S.M.; Woo, J.H.; Klimont, Z. A technology-based global inventory of black and organic carbon emissions from combustion. J. Geophys. Res. Atmos. 2004, 109. [CrossRef]

55. Dumont, M.; Brun, E.; Picard, G.; Michou, M.; Libois, Q.; Petit, J.; Geyer, M.; Morin, S.; Josse, B. Contribution of light-absorbing impurities in snow to Greenland's darkening since 2009. Nat. Geosci. 2014, 7, 509-512. [CrossRef]

56. Flanner, M.G.; Zender, C.S.; Randerson, J.T.; Rasch, P.J. Present-day climate forcing and response from black carbon in snow. J. Geophys. Res. Atmos. 2007, 112, D11202. [CrossRef]

57. Lau, W.K.; Kim, M.-K.; Kim, K.-M.; Lee, W.-S. Enhanced surface warming and accelerated snow melt in the Himalayas and Tibetan Plateau induced by absorbing aerosols. Environ. Res. Lett. 2010, 5, 25204. [CrossRef]

58. Yasunari, T.; Bonasoni, P.; Laj, P.; Fujita, K.; Vuillermoz, E.; Marinoni, A.; Cristofanelli, P.; Duchi, R.; Tartari, G.; Lau, K.-M. Estimated impact of black carbon deposition during pre-monsoon season from Nepal Climate Observatory-Pyramid data and snow albedo changes over Himalayan glaciers. Atmos. Chem. Phys. 2010, 10, 6603-6615. [CrossRef]

59. Gustafsson, Ö.; Ramanathan, V. Convergence on climate warming by black carbon aerosols. Proc. Natl. Acad. Sci. USA 2016, 113, 4243-4245. [CrossRef]

60. Ji, Z.; Kang, S.C.; Cong, Z.Y.; Zhang, Q.G.; Yao, T.D. 2015: Simulation of carbonaceous aerosols over the Third Pole and adjacent regions: Distribution, transportation, deposition, and climatic effects. Clim. Dyn. 2015, 45, 2831-2846. [CrossRef]

61. Qian, Y.; Yasunari, T.J.; Doherty, S.J.; Flanner, M.G.; Lau, W.K.; Ming, J.; Wang, H.; Wang, M.; Warren, S.G.; Zhang, R. Lightabsorbing particles in snow and ice: Measurement and modeling of climatic and hydrological impact. Adv. Atmos. Sci. 2015, 32, 64-91. [CrossRef]

62. Wild, M.; Folini, D.; Schär, C.; Loeb, N.; König-Langlo, G. Earth Radiation Balance as Observed and Represented in CMIP5 models. In Proceedings of the EGU General Assembly Conference Abstracts, Vienna, Austria, 27 April-2 May $2014 ;$ p. 7867.

63. Xu, Y.; Ramanathan, V.; Washington, W. Observed high-altitude warming and snow cover retreat over Tibet and the Himalayas enhanced by black carbon aerosols. Atmos. Chem. Phys. 2016, 16, 1303-1315. [CrossRef]

64. Yasunari, T.J.; Tan, Q.; Lau, K.-M.; Bonasoni, P.; Marinoni, A.; Laj, P.; Ménégoz, M.; Takemura, T.; Chin, M. Estimated range of black carbon dry deposition and the related snow albedo reduction over Himalayan glaciers during dry pre-monsoon periods. Atmos. Environ. 2013, 78, 259-267. [CrossRef] 
65. Fujita, K.-I.; Maeda, D.; Xiao, Q.; Srinivasula, S.M. Nrf2-mediated induction of p62 controls Toll-like receptor-4-driven aggresomelike induced structure formation and autophagic degradation. Proc. Natl. Acad. Sci. USA 2011, 108, 1427-1432. [CrossRef]

66. Benning, L.G.; Anesio, A.M.; Lutz, S.; Tranter, M. Biological impact on Greenland's albedo. Nat. Geosci. 2014, 7, 691. [CrossRef]

67. Uetake, J.; Naganuma, T.; Hebsgaard, M.B.; Kanda, H.; Kohshima, S. Communities of algae and cyanobacteria on glaciers in west Greenland. Polar. Sci. 2010, 4, 71-80. [CrossRef]

68. Yallop, M.L.; Anesio, A.M.; Perkins, R.G.; Cook, J.; Telling, J.; Fagan, D.; MacFarlane, J.; Stibal, M.; Barker, G.; Bellas, C. Photophysiology and albedo-changing potential of the ice algal community on the surface of the Greenland ice sheet. ISME J. 2012, 6, 2302-2313. [CrossRef] 